\title{
A Nonhomogeneous Dirichlet Problem for a Nonlinear Pseudoparabolic Equation Arising in the Flow of Second-Grade Fluid
}

\author{
Le Thi Phuong Ngoc, ${ }^{1}$ Truong Thi Nhan, ${ }^{2,3}$ and Nguyen Thanh Long ${ }^{3}$ \\ ${ }^{1}$ University of Khanh Hoa, 01 Nguyen Chanh Str., Nha Trang, Vietnam \\ ${ }^{2}$ The Faculty of Natural Basic Sciences, Vietnamese Naval Academy, 30 Tran Phu Street, Nha Trang, Vietnam \\ ${ }^{3}$ Department of Mathematics and Computer Science, University of Natural Science, Vietnam National University Ho Chi Minh City, \\ 227 Nguyen Van Cu Str., Dist. 5, Ho Chi Minh City, Vietnam
}

Correspondence should be addressed to Nguyen Thanh Long; longnt2@gmail.com

Received 18 September 2016; Accepted 13 November 2016

Academic Editor: Andrew Pickering

Copyright (C) 2016 Le Thi Phuong Ngoc et al. This is an open access article distributed under the Creative Commons Attribution License, which permits unrestricted use, distribution, and reproduction in any medium, provided the original work is properly cited.

\begin{abstract}
We study the following initial-boundary value problem $\left\{u_{t}-(\mu(t)+\alpha(t)(\partial / \partial t))\left(\partial^{2} u / \partial x^{2}+(\gamma / x)(\partial u / \partial x)\right)+f(u)=f_{1}(x, t), 1<x<R\right.$, $\left.t>0 ; u(1, t)=g_{1}(t), u(R, t)=g_{R}(t) ; u(x, 0)=\widetilde{u}_{0}(x)\right\}$, where $\gamma>0, R>1$ are given constants and $f, f_{1}, g_{1}, g_{R}, \widetilde{u}_{0}, \alpha$, and $\mu$ are given functions. In Part 1 , we use the Galerkin method and compactness method to prove the existence of a unique weak solution of the problem above on $(0, T)$, for every $T>0$. In Part 2, we investigate asymptotic behavior of the solution as $t \rightarrow+\infty$. In Part 3 , we prove the existence and uniqueness of a weak solution of problem $\left\{u_{t}-(\mu(t)+\alpha(t)(\partial / \partial t))\left(\partial^{2} u / \partial x^{2}+(\gamma / x)(\partial u / \partial x)\right)+f(u)=\right.$ $\left.f_{1}(x, t), 1<x<R, t>0 ; u(1, t)=g_{1}(t), u(R, t)=g_{R}(t)\right\}$ associated with a " $\left.\eta, T\right)$-periodic condition" $u(x, 0)=\eta u(x, T)$, where $0<|\eta| \leq 1$ is given constant.
\end{abstract}

\section{Introduction}

In this paper, we consider the following nonlinear pseudoparabolic equation:

$$
\begin{aligned}
u_{t} & -\left(\mu(t)+\alpha(t) \frac{\partial}{\partial t}\right)\left(\frac{\partial^{2} u}{\partial x^{2}}+\frac{\gamma}{x} \frac{\partial u}{\partial x}\right)+f(u) \\
= & f_{1}(x, t), \quad 1<x<R, t>0,
\end{aligned}
$$

associated with the boundary conditions

$$
\begin{gathered}
u(1, t)=g_{1}(t), \\
u(R, t)=g_{R}(t)
\end{gathered}
$$

and the initial condition

$$
u(x, 0)=\widetilde{u}_{0}(x),
$$

or the " $\eta, T)$-periodic condition"

$$
u(x, 0)=\eta u(x, T),
$$

where $\gamma>0, R>1$, and $0<|\eta| \leq 1$ are given constants and $f, f_{1}, g_{1}, g_{R}, \widetilde{u}_{0}, \alpha$, and $\mu$ are given functions satisfying conditions specified later.

In the case of $\gamma=1, \mu(t)=\mu>0$, and $\alpha(t)=\alpha>0$ being the constants, the initial-boundary value problems (1)(3) are classical and have a long history of applications and mathematical development. We refer to the monographs of Al'shin et al. [1] and of Carroll and Showalter [2] for references and results on pseudoparabolic or Sobolev type equations. We also refer to [3] for asymptotic behavior and to[4] for nonlinear problems. Problems of this type arise in material science and physics, which have been extensively studied, and several results concerning existence, regularity, and asymptotic behavior have been established.

Equation (1) arises within frameworks of mathematical models in engineering and physical sciences (see [5-11] for references therein and interesting results on second grade fluids or a fourth grade fluid or other unsteady flows). It is well known that fluid solid mixtures are generally considered 
as second-grade fluids and are modeled as fluids with variable physical parameters; thus, an analysis is performed for a second-grade fluid with space dependent viscosity, elasticity, and density.

In [9], some unsteady flow problems of a second-grade fluid were considered. The flows are generated by the sudden application of a constant pressure gradient or by the impulsive motion of a boundary. Here, the velocities of the flows are described by the partial differential equations and exact analytic solutions of these differential equations are obtained. Suppose that the second-grade fluid is in a circular cylinder and is initially at rest, and the fluid starts suddenly due to the motion of the cylinder parallel to its length. The axis of the cylinder is chosen as the $z$-axis. Using cylindrical polar coordinates, the governing partial differential equation is

$$
\begin{aligned}
\frac{\partial w}{\partial t}=\left(v+\alpha \frac{\partial}{\partial t}\right)\left(\frac{\partial^{2}}{\partial r^{2}}+\frac{1}{r} \frac{\partial}{\partial r}\right) & w(r, t)-N w, \\
w(a, t) & =W, \quad t>0, \\
w(r, 0) & =0, \quad 0 \leq r<a, t>0,
\end{aligned}
$$

where $w(r, t)$ is the velocity along the $z$-axis, $v$ is the kinematic viscosity, $\alpha$ is the material parameter, and $N$ is the imposed magnetic field. In the boundary and initial conditions, $W$ is the constant velocity at $r=a$ and $a$ is the radius of the cylinder.

In [6], two types of time-dependent flows were investigated. An eigenfunction expansion method was used to find the velocity distribution. The obtained solutions satisfy the boundary and initial conditions and the governing equation. Remarkably, some exact analytic solutions are possible for flows involving second-grade fluid with variable material properties in terms of trigonometric and Chebyshev functions.

In [5], Mahmood et al. have considered the longitudinal oscillatory motion of second-grade fluid between two infinite coaxial circular cylinders, oscillating along their common axis with given constant angular frequencies $\Omega_{1}$ and $\Omega_{2}$. Velocity field and associated tangential stress of the motion were determined by using Laplace and Hankel transforms. In order to find exact analytic solutions for the flow of secondgrade fluid between two longitudinally oscillating cylinders, the following problem was studied:

$$
\begin{aligned}
\frac{\partial v}{\partial t} & =\left(\mu+\alpha \frac{\partial}{\partial t}\right)\left(\frac{\partial^{2}}{\partial r^{2}}+\frac{1}{r} \frac{\partial}{\partial r}\right) v(r, t), \\
R_{1}<r<R_{2}, t>0, & \\
v\left(R_{1}, t\right) & =V_{1} \sin \left(\Omega_{1} t\right), \\
v\left(R_{2}, t\right) & =V_{2} \sin \left(\Omega_{2} t\right), \\
v(r, 0) & =0, \quad R_{1} \leq r \leq R_{2},
\end{aligned}
$$

where $0<R_{1}<R_{2}, \mu, \alpha, V_{1}, V_{2}, \Omega_{1}$, and $\Omega_{2}$ are positive constants. The solutions obtained have been presented under series form in terms of Bessel functions $J_{0}(x), Y_{0}(x), J_{1}(x)$, $Y_{1}(x), J_{2}(x)$, and $Y_{2}(x)$, satisfying the governing equation and all imposed initial and boundary conditions.

The nonlinear parabolic problems of the form (1)-(3), with/without the term $u_{r r}+(\gamma / r) u_{r}$, were also studied in $[12,13]$ and references therein. In [12], by using the Galerkin and compactness method in appropriate Sobolev spaces with weight, the authors proved the existence of a unique weak solution of the following initial and boundary value problem for nonlinear parabolic equation:

$$
\begin{aligned}
u_{t}-a(t)\left(u_{r r}+\frac{\gamma}{r} u_{r}\right)+F(r, u) & =f(r, t), \\
& 0<r<1,0<t<T, \\
\left|\lim _{r \rightarrow 0_{+}} r^{\gamma / 2} u_{r}(r, t)\right| & <+\infty, \\
u_{r}(1, t)+h(t)\left(u(1, t)-\bar{u}_{0}\right) & =0, \\
u(r, 0) & =u_{0}(r) .
\end{aligned}
$$

Furthermore, asymptotic behavior of the solution as $t \rightarrow+\infty$ was studied. In [13], the following nonlinear heat equation associated with Dirichlet-Robin conditions was investigated:

$$
\begin{aligned}
& u_{t}-\frac{\partial}{\partial x}\left[\mu(x, t) u_{x}\right]+f(u)=f_{1}(x, t), \\
&(x, t) \in(0,1) \times(0, T), \\
& u_{x}(0, t)=h_{0} u(0, t)+g_{0}(t), \\
&-u_{x}(1, t)=h_{1} u(1, t)+g_{1}(t), \\
& u(x, 0)=u_{0}(x) .
\end{aligned}
$$

Condition (4), which we call " $(\eta, T)$-periodic condition," is known as a drifted periodic condition (see [14]). Indeed, if $u(t)=\eta u(t+T), \forall t \geq 0$, in the case of $0<|\eta| \leq 1$, then we have

$$
u(t+T)=\frac{1}{\eta} u(t)=u(t)+\left(\frac{1}{\eta}-1\right) u(t), \quad \forall t \geq 0,
$$

which means

$$
u(t+T)=u(t)+\delta(t), \quad \forall t \geq 0,
$$

with $\delta(t)=(1 / \eta-1) u(t)$ satisfying the condition

$$
\delta(t)=\eta \delta(t+T), \quad \forall t \geq 0 .
$$

Note that (11) holds by the fact that

$$
\begin{aligned}
\eta \delta(t+T) & =\eta\left[\left(\frac{1}{\eta}-1\right) u(t+T)\right]=\left(\frac{1}{\eta}-1\right) u(t) \\
& =\delta(t), \quad \forall t \geq 0 .
\end{aligned}
$$

With $\eta=1$, (4) leads to $T$-periodic condition

$$
u(x, 0)=u(x, T),
$$


and with $\eta=-1$, we have the antiperiodic condition

$$
u(x, 0)=-u(x, T)
$$

The present paper is concerned with the second-grade fluid in a circular cylinder associated with the initial condition (3) or a drifted periodic condition (10). The extensive study of such flows is motivated by both their fundamental interest and their practical importance (see [9]).

This paper is a continuation of paper [15] dealing with the nonlinear pseudoparabolic equation (1) associated with the mixed inhomogeneous condition, in the case of $\gamma=1$, $\mu(t)=\mu>0, \alpha(t)=\alpha>0$ being the constants. It consists of five sections. First, preliminaries are done in Section 2. Under appropriate conditions, the existence of a unique weak solution of problems (1)-(3) is proved in Section 3. Next, an asymptotic behavior of the solution of problems (1)-(3), as $t \rightarrow+\infty$, is discussed in Section 4. Finally, Section 5 is devoted to the establishment, the existence, and uniqueness of a weak solution of problems (1), (2), and (4).

Because of mathematical context, the results obtained here generalize relatively the ones in $[12,13,15]$, by improving the techniques used as before and with appropriate modifications.

\section{Preliminaries}

Put $\Omega=(1, R), Q_{T}=\Omega \times(0, T), T>0$. We omit the definitions of the usual function spaces: $C^{m}(\bar{\Omega}), L^{p}(\Omega)$, and $W^{m, p}(\Omega)$. We define $W^{m, p}=W^{m, p}(\Omega), L^{p}=W^{0, p}(\Omega)$ and $H^{m}=W^{m, 2}(\Omega), 1 \leq p \leq \infty, m=0,1, \ldots$. The norm in $L^{2}$ is denoted by $\|\cdot\|$. We also denote by $(\cdot, \cdot)$ the scalar product in $L^{2}$. We denote by $\|\cdot\|_{X}$ the norm of a Banach space $X$ and by $X^{\prime}$ the dual space of $X$. We denote by $L^{p}(0, T ; X) 1 \leq p \leq \infty$ for the Banach space of the real functions $u:(0, T) \rightarrow X$ measurable, such that

$$
\begin{aligned}
& \|u\|_{L^{p}(0, T ; X)}=\left(\int_{0}^{T}\|u(t)\|_{X}^{p} d t\right)^{1 / p}<\infty \\
& \text { for } 1 \leq p<\infty
\end{aligned}
$$

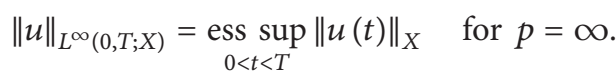

Let $u(t), u^{\prime}(t)=u_{t}(t), u^{\prime \prime}(t)=u_{t t}(t), u_{x}(t)$, and $u_{x x}(t)$ denote $u(x, t),(\partial u / \partial t)(x, t),\left(\partial^{2} u / \partial t^{2}\right)(x, t),(\partial u / \partial x)(x, t)$, $\left(\partial^{2} u / \partial x^{2}\right)(x, t)$, respectively.

On $H^{1}$, we shall use the following norm:

$$
\|v\|_{H^{1}}=\left(\|v\|^{2}+\left\|v_{x}\right\|^{2}\right)^{1 / 2}
$$

We put

$$
H_{0}^{1}=\left\{v \in H^{1}(\Omega): v(1)=v(R)=0\right\} .
$$

$H_{0}^{1}$ is a closed subspace of $H^{1}$ and on $H_{0}^{1}$, two norms $\|v\|_{H^{1}}$ and $\left\|v_{x}\right\|$ are equivalent.
Note that $L^{2}$ and $H^{1}$ are also the Hilbert spaces with respect to the corresponding scalar products

$$
\langle u, v\rangle=\int_{1}^{R} x^{\gamma} u(x) v(x) d x, \quad\langle u, v\rangle+\left\langle u_{x}, v_{x}\right\rangle,
$$

respectively. The norms in $L^{2}$ and $H^{1}$ induced by the corresponding scalar products are denoted by $\|\cdot\|_{0}$ and $\|\cdot\|_{1}$, respectively. $H_{0}^{1}$ is continuously and densely embedded in $L^{2}$. Identifying $L^{2}$ with $\left(L^{2}\right)^{\prime}$ (the dual of $L^{2}$ ), we have $H_{0}^{1} \hookrightarrow$ $L^{2} \hookrightarrow\left(H_{0}^{1}\right)^{\prime}=H^{-1}$; on the other hand, the notation $\langle\cdot, \cdot\rangle$ is used for the pairing between $H_{0}^{1}$ and $H^{-1}$.

We then have the following lemmas, the proofs of which can be found in [16].

Lemma 1. We have the following inequalities:

(i) $\|v\| \leq\|v\|_{0} \leq \sqrt{R^{\gamma}}\|v\|, \quad \forall v \in L^{2}$,

(ii) $\|v\|_{H^{1}} \leq\|v\|_{1} \leq \sqrt{R^{\gamma}}\|v\|_{H^{1}}, \quad \forall v \in H^{1}$.

Lemma 2. The imbedding $H^{1} \hookrightarrow C^{0}(\bar{\Omega})$ is compact.

Lemma 3. The imbedding $H_{0}^{1} \hookrightarrow C^{0}(\bar{\Omega})$ is compact and

(i) $\|v\|_{C^{0}(\bar{\Omega})} \leq \sqrt{R-1}\left\|v_{x}\right\| \quad \forall v \in H_{0}^{1}$,

(ii) $\|v\| \leq \frac{R-1}{\sqrt{2}}\left\|v_{x}\right\| \quad \forall v \in H_{0}^{1}$,

(iii) $\|v\|_{0} \leq \sqrt{\frac{R^{\gamma}}{2}}(R-1)\left\|v_{x}\right\|_{0} \quad \forall v \in H_{0}^{1}$.

Remark 4. On $L^{2}$, two norms $v \mapsto\|v\|$ and $v \mapsto\|v\|_{0}$ are equivalent. So there are two norms $v \mapsto\|v\|_{H^{1}}$ and $v \mapsto\|v\|_{1}$ on $H^{1}$ and four norms $v \mapsto\|v\|_{H^{1}}, v \mapsto\|v\|_{1}, v \mapsto\left\|v_{x}\right\|$, and $v \mapsto\left\|v_{x}\right\|_{0}$ on $H_{0}^{1}$.

Consider $a(\cdot, \cdot)$ is the symmetric bilinear form on $H_{0}^{1} \times H_{0}^{1}$ defined by

$$
a(u, w)=\left\langle u_{x}, w_{x}\right\rangle, \quad \forall u, w \in H_{0}^{1} .
$$

Then, the symmetric bilinear form $a(\cdot, \cdot)$ is continuous on $H_{0}^{1} \times H_{0}^{1}$ and coercive on $H_{0}^{1}$.

We have also the following lemma.

Lemma 5. There exists the Hilbert orthonormal base $\left\{w_{j}\right\}$ of $L^{2}$ consisting of the eigenfunctions $w_{j}$ corresponding to the eigenvalue $\bar{\lambda}_{j}$ such that

$$
0<\bar{\lambda}_{1} \leq \bar{\lambda}_{2} \leq \cdots \leq \bar{\lambda}_{j} \leq \bar{\lambda}_{j+1} \leq \cdots,
$$

$\lim _{j \rightarrow+\infty} \bar{\lambda}_{j}=+\infty$

$a\left(w_{j}, w\right)=\bar{\lambda}_{j}\left\langle w_{j}, w\right\rangle \quad \forall w \in H_{0}^{1}, j=1,2, \ldots$

Furthermore, the sequence $\left\{w_{j} / \sqrt{\overline{\bar{\lambda}}_{j}}\right\}$ is also the Hilbert orthonormal base of $H_{0}^{1}$ with respect to the scalar product $a(\cdot, \cdot)$. 
On the other hand, we also have $w_{j}$ satisfying the following boundary value problem:

$$
\begin{aligned}
-\left(w_{j x x}+\frac{\gamma}{x} w_{j x}\right) & =\bar{\lambda}_{j} w_{j}, \quad \text { in }(1, R), \\
w_{j}(1) & =w_{j}(R)=0, \quad w_{j} \in C^{\infty}([1, R]) .
\end{aligned}
$$

The proof of Lemma 5 can be found in $[17$, p. 87 , Theorem 7.7], with $H=L^{2}$ and $V=H_{0}^{1}$ and $a(\cdot, \cdot)$ as defined by (21).

\section{The Existence and the Uniqueness}

Now, we consider problems (1)-(3) in which $\gamma$ is a positive constant and make the following assumptions:

$\left(H_{1}\right) \widetilde{u}_{0} \in H^{1}$.

$\left(H_{2}\right) g_{1}, g_{R} \in W^{1,1}(0, T), \widetilde{u}_{0}(1)-g_{1}(0)=\widetilde{u}_{0}(R)-g_{R}(0)=0$.

$\left(H_{3}\right) \alpha \in W^{1,1}(0, T), \alpha(t) \geq \alpha_{*}>0, \forall t \in[0, T]$.

$\left(H_{4}\right) \mu \in W^{1,1}(0, T), \mu(t) \geq \mu_{*}>0, \forall t \in[0, T]$.

$\left(H_{5}\right) f_{1} \in L^{1}\left(0, T ; L^{2}\right)$.

$\left(H_{6}\right) f \in C^{0}(\mathbb{R} ; \mathbb{R})$ satisfies the condition that there exists positive constant $\delta$ such that $(y-z)(f(y)-f(z)) \geq$ $-\delta|y-z|^{2}$, for all $y, z \in \mathbb{R}$.

In case $g_{1} \neq 0$ or $g_{R} \neq 0$, it is clearly that problems (1)-(3) reduce to a problem with homogeneous boundary conditions by the suitable transformation. Indeed, putting $\varphi(x, t)=((x-1) /(R-1)) g_{R}(t)+((R-x) /(R-1)) g_{1}(t)$, by the transformation $v(x, t)=u(x, t)-\varphi(x, t)$, problems $(1)-(3)$ reduce to the following problem:

$$
\begin{aligned}
& v_{t}-\left(\mu(t)+\alpha(t) \frac{\partial}{\partial t}\right)\left(\frac{\partial^{2} v}{\partial x^{2}}+\frac{\gamma}{x} \frac{\partial v}{\partial x}\right)+f(v+\varphi) \\
& =\bar{f}_{1}(x, t), \quad 1<x<R, t>0, \\
& v(1, t)=v(R, t)=0, \\
& v(x, 0)=\widetilde{v}_{0}(x),
\end{aligned}
$$

where

$$
\begin{aligned}
& \bar{f}_{1}(x, t)=f_{1}(x, t)-\frac{1}{R-1}\left[(x-1) g_{R}^{\prime}(t)\right. \\
& \left.\quad+(R-x) g_{1}^{\prime}(t)\right] \\
& \quad+\frac{\gamma}{(R-1) x}\left[\mu(t)\left(g_{R}(t)-g_{1}(t)\right)\right. \\
& \left.\quad+\alpha(t)\left(g_{R}^{\prime}(t)-g_{1}^{\prime}(t)\right)\right], \\
& \widetilde{v}_{0}(x)=\widetilde{u}_{0}(x)-\varphi(x, 0), \quad \widetilde{v}_{0} \in H_{0}^{1}
\end{aligned}
$$

and $\tilde{u}_{0}, g_{1}$, and $g_{R}$ satisfy the condition $\tilde{u}_{0}(1)-g_{1}(0)=\tilde{u}_{0}(R)-$ $g_{R}(0)=0$.

The weak formulation of the initial-boundary value problem (24) can be given in the following manner: Find $v \in L^{\infty}\left(0, T ; H_{0}^{1}\right)$ with $t v_{t} \in L^{2}\left(0, T ; H_{0}^{1}\right)$, such that $v$ satisfies the following variational equation:

$$
\begin{aligned}
& \frac{d}{d t}[\langle v(t), w\rangle+\alpha(t) a(v(t), w)] \\
& +\left(\mu(t)-\alpha^{\prime}(t)\right) a(v(t), w) \\
& +\langle f(v(t)+\varphi(t)), w\rangle=\left\langle\bar{f}_{1}(t), w\right\rangle, \\
& \forall w \in H_{0}^{1}, \text { a.e., } t \in(0, T), \\
& v(0)=\widetilde{v}_{0},
\end{aligned}
$$

where $a(\cdot, \cdot)$ is the symmetric bilinear form on $H_{0}^{1} \times H_{0}^{1}$ defined by (21).

Then, we have the following theorem.

Theorem 6. Let $T>0$ and $\left(H_{1}\right)-\left(H_{6}\right)$ hold. Then, problem (24) has a unique weak solution $v$ such that

$$
\begin{gathered}
v \in L^{\infty}\left(0, T ; H_{0}^{1}\right), \\
t v_{t} \in L^{2}\left(0, T ; H_{0}^{1}\right) .
\end{gathered}
$$

Moreover, if $\left(H_{5}\right)$ is replaced by $f_{1} \in L^{2}\left(Q_{T}\right)$, then the solution $v$ satisfies

$$
\begin{aligned}
& v \in L^{\infty}\left(0, T ; H_{0}^{1}\right), \\
& v_{t} \in L^{2}\left(0, T ; H_{0}^{1}\right) .
\end{aligned}
$$

Proof. The proof consists of several steps.

Step 1 (the Faedo-Galerkin approximation (introduced by Lions [18])). Consider the basis $\left\{w_{j}\right\}$ for $H_{0}^{1}$ as in Lemma 5. We find the approximate solution of problem (24) in the form

$$
v_{m}(t)=\sum_{j=1}^{m} c_{m j}(t) w_{j}
$$

where the coefficients $c_{m j}$ satisfy the system of linear differential equations

$$
\begin{aligned}
& \left\langle v_{m}^{\prime}(t), w_{j}\right\rangle+\alpha(t) a\left(v_{m}^{\prime}(t), w_{j}\right) \\
& +\mu(t) a\left(v_{m}(t), w_{j}\right) \\
& +\left\langle f\left(v_{m}(t)+\varphi(t)\right), w_{j}\right\rangle=\left\langle\bar{f}_{1}(t), w_{j}\right\rangle, \\
& \quad 1 \leq j \leq m, \\
& v_{m}(0)=v_{0 m},
\end{aligned}
$$

where

$$
v_{0 m}=\sum_{j=1}^{m} \alpha_{m j} w_{j} \longrightarrow \widetilde{v}_{0} \quad \text { strongly in } H_{0}^{1} .
$$


The system of (30) can be rewritten in the form

$$
\begin{aligned}
& c_{m j}^{\prime}(t)+\frac{\bar{\lambda}_{j} \mu(t)}{1+\bar{\lambda}_{j} \alpha(t)} c_{m j}(t) \\
& +\frac{1}{1+\bar{\lambda}_{j} \alpha(t)}\left\langle f\left(v_{m}(t)+\varphi(t)\right), w_{j}\right\rangle \\
& =\frac{1}{1+\bar{\lambda}_{j} \alpha(t)}\left\langle\bar{f}_{1}(t), w_{j}\right\rangle, \\
& c_{m j}(0)=\alpha_{m j}, \quad 1 \leq j \leq m .
\end{aligned}
$$

It is clear that for each $m$ there exists a solution $v_{m}(t)$ in the form of (29) which satisfies (30) almost everywhere on $0 \leq t \leq \widetilde{T}_{m}$ for some $\widetilde{T}_{m}, 0<\widetilde{T}_{m} \leq T$. The following estimates allow one to take $\widetilde{T}_{m}=T$ for all $m$.

Step 2 (a priori estimates)

(a) The First Estimate. Multiplying the $j$ th equation of (30) by $c_{m j}(t)$ and summing up with respect to $j$, afterwards, integrating by parts with respect to the time variable from 0 to $t$, we get after some rearrangements:

$$
\begin{aligned}
& \left\|v_{m}(t)\right\|_{0}^{2}+\alpha(t)\left\|v_{m x}(t)\right\|_{0}^{2} \\
& =\left\|v_{0 m}\right\|_{0}^{2}+\alpha(0)\left\|v_{0 m x}\right\|_{0}^{2} \\
& \quad-\int_{0}^{t}\left(2 \mu(s)-\alpha^{\prime}(s)\right)\left\|v_{m x}(s)\right\|_{0}^{2} d s \\
& \quad-2 \int_{0}^{t}\left\langle f\left(v_{m}(s)+\varphi(s)\right), v_{m}(s)\right\rangle d s \\
& \quad+2 \int_{0}^{t}\left\langle\bar{f}_{1}(s), v_{m}(s)\right\rangle d s .
\end{aligned}
$$

By $v_{0 m} \rightarrow \widetilde{v}_{0}$ strongly in $H_{0}^{1}$, we have

$$
\left\|v_{0 m}\right\|_{0}^{2}+\alpha(0)\left\|v_{0 m x}\right\|_{0}^{2} \leq \bar{S}_{0}, \quad \forall m,
$$

where $\bar{S}_{0}$ always indicates a bound depending on $\widetilde{v}_{0}$.

Put

$$
S_{m}(t)=\left\|v_{m}(t)\right\|_{0}^{2}+\alpha_{*}\left\|v_{m x}(t)\right\|_{0}^{2}
$$

By the assumptions $\left(H_{3}\right)-\left(H_{6}\right)$, we estimate without difficulty the following terms in (33) as follows:

$$
\begin{gathered}
-\int_{0}^{t}\left(2 \mu(s)-\alpha^{\prime}(s)\right)\left\|v_{m x}(s)\right\|_{0}^{2} d s \\
\leq \frac{1}{\alpha_{*}} \int_{0}^{t}\left|2 \mu(s)-\alpha^{\prime}(s)\right| S_{m}(s) d s ;
\end{gathered}
$$

$$
\begin{aligned}
& -2 \int_{0}^{t}\left\langle f\left(v_{m}(s)+\varphi(s)\right), v_{m}(s)\right\rangle d s \\
& \leq 2 \delta \int_{0}^{t}\left\|v_{m}(s)\right\|_{0}^{2} d s \\
& \quad+2 \int_{0}^{t}\|f(\varphi(s))\|_{0}\left\|v_{m}(s)\right\|_{0} d s \\
& \leq(2 \delta+1) \int_{0}^{t}\left\|v_{m}(s)\right\|_{0}^{2} d s+\int_{0}^{T}\|f(\varphi(s))\|_{0}^{2} d s ; \\
& 2 \int_{0}^{t}\left\langle\bar{f}_{1}(s), v_{m}(s)\right\rangle d s \\
& \leq\left\|\bar{f}_{1}\right\|_{L^{1}\left(0, T ; L^{2}\right)}+\int_{0}^{t}\left\|\bar{f}_{1}(s)\right\|_{0}\left\|v_{m}(s)\right\|_{0}^{2} d s \\
& \leq\left\|\bar{f}_{1}\right\|_{L^{1}\left(0, T ; L^{2}\right)}+\int_{0}^{t}\left\|\bar{f}_{1}(s)\right\|_{0} S_{m}(s) d s .
\end{aligned}
$$

Hence, it follows from (33), (34), and (36) that

$$
S_{m}(t) \leq C_{T}^{(1)}+\int_{0}^{t} d_{T}^{(1)}(s) S_{m}(s) d s
$$

where

$$
\begin{gathered}
C_{T}^{(1)}=\bar{S}_{0}+\left\|\bar{f}_{1}\right\|_{L^{1}\left(0, T ; L^{2}\right)}+\int_{0}^{T}\|f(\varphi(s))\|_{0}^{2} d s, \\
d_{T}^{(1)}(s)=1+2 \delta+\left\|\bar{f}_{1}(s)\right\|_{0}+\frac{1}{\alpha_{*}}\left|2 \mu(s)-\alpha^{\prime}(s)\right|, \\
d_{T}^{(1)} \in L^{1}(0, T) .
\end{gathered}
$$

By Gronwall's lemma, we obtain from (37) that

$$
S_{m}(t) \leq C_{T}^{(1)} \exp \left(\int_{0}^{t} d_{T}^{(1)}(s) d s\right) \leq C_{T}
$$

for all $m \in \mathbb{N}$, for all $t, 0 \leq t \leq \widetilde{T}_{m} \leq T$; that is, $\widetilde{T}_{m}=T$, where $C_{T}$ always indicates a bound depending on $T$.

(b) The Second Estimate. Multiplying the $j$ th equation of (30) by $2 t^{2} c_{m j}^{\prime}(t)$ and summing up with respect to $j$, we have

$$
\begin{aligned}
2\left\|t v_{m}^{\prime}(t)\right\|_{0}^{2}+2 \alpha(t)\left\|t v_{m x}^{\prime}(t)\right\|_{0}^{2} \\
+\frac{d}{d t}\left[\mu(t)\left\|t v_{m x}(t)\right\|_{0}^{2}\right] \\
=\left(t^{2} \mu(t)\right)^{\prime}\left\|v_{m x}(t)\right\|_{0}^{2} \\
\quad-2 t^{2}\left\langle f\left(v_{m}(t)+\varphi(t)\right), v_{m}^{\prime}(t)\right\rangle \\
+2 t^{2}\left\langle\bar{f}_{1}(t), v_{m}^{\prime}(t)\right\rangle .
\end{aligned}
$$


Integrating (40), we get

$$
\begin{aligned}
2 \int_{0}^{t} & \left\|s v_{m}^{\prime}(s)\right\|_{0}^{2} d s+2 \int_{0}^{t} \alpha(s)\left\|s v_{m x}^{\prime}(s)\right\|_{0}^{2} d s \\
& +\mu(t)\left\|t v_{m x}(t)\right\|_{0}^{2} \\
= & \int_{0}^{t}\left(s^{2} \mu(s)\right)^{\prime}\left\|v_{m x}(s)\right\|_{0}^{2} d s \\
& -2 \int_{0}^{t}\left\langle s f\left(v_{m}(s)+\varphi(s)\right), s v_{m}^{\prime}(s)\right\rangle d s \\
& +2 \int_{0}^{t}\left\langle s \bar{f}_{1}(s), s v_{m}^{\prime}(s)\right\rangle d s .
\end{aligned}
$$

We shall estimate the terms of (41) as follows:

$$
\begin{aligned}
& \int_{0}^{t}\left(s^{2} \mu(s)\right)^{\prime}\left\|v_{m x}(s)\right\|_{0}^{2} d s \\
& \leq \frac{1}{\alpha_{*}} \int_{0}^{t}\left|\left(s^{2} \mu(s)\right)^{\prime}\right| S_{m}(s) d s \\
& \leq \frac{C_{T}}{\alpha_{*}} \int_{0}^{T}\left|\left(s^{2} \mu(s)\right)^{\prime}\right| d s ; \\
& 2 \int_{0}^{t}\left\langle s \bar{f}_{1}(s), s v_{m}^{\prime}(s)\right\rangle d s \\
& \leq 2 \int_{0}^{T}\left\|s \bar{f}_{1}(s)\right\|_{0}^{2} d s+\frac{1}{2} \int_{0}^{t}\left\|s v_{m}^{\prime}(s)\right\|_{0}^{2} d s .
\end{aligned}
$$

On the other hand, we have

$$
\begin{aligned}
\left|v_{m}(x, s)\right|+|\varphi(x, s)| \leq & \left\|v_{m}(s)\right\|_{C^{0}(\bar{\Omega})}+\left|g_{1}(s)\right| \\
& +\left|g_{R}(s)\right| \\
\leq & \sqrt{\frac{(R-1) C_{T}}{\alpha_{*}}}+\left\|g_{1}\right\|_{C^{0}([0, T])} \\
& +\left\|g_{R}\right\|_{C^{0}([0, T])} \equiv \bar{C}_{T},
\end{aligned}
$$

and hence

$$
\begin{gathered}
2 \int_{0}^{t}\left\langle s f\left(v_{m}(s)+\varphi(s)\right), s v_{m}^{\prime}(s)\right\rangle d s \\
\leq 2 \int_{0}^{t}\left\|s f\left(v_{m}(s)+\varphi(s)\right)\right\|_{0}^{2} d s \\
\quad+\frac{1}{2} \int_{0}^{t}\left\|s v_{m}^{\prime}(s)\right\|_{0}^{2} d s \\
\leq 2 \int_{0}^{t} s^{2} d s \int_{1}^{R} x^{\gamma} \sup _{|z| \leq \bar{C}_{T}} f^{2}(z) d x \\
+\frac{1}{2} \int_{0}^{t}\left\|s v_{m}^{\prime}(s)\right\|_{0}^{2} d s
\end{gathered}
$$

$$
\begin{aligned}
\leq & 2 T^{2}\left(\frac{R^{\gamma+1}-1}{\gamma+1}\right) \sup _{|z| \leq \bar{C}_{T}} f^{2}(z) d x \\
& +\frac{1}{2} \int_{0}^{t}\left\|s v_{m}^{\prime}(s)\right\|_{0}^{2} d s .
\end{aligned}
$$

It follows from (41)-(43) and (45) that

$$
\begin{aligned}
& \int_{0}^{t}\left\|s v_{m}^{\prime}(s)\right\|_{0}^{2} d s+2 \alpha_{*} \int_{0}^{t}\left\|s v_{m x}^{\prime}(s)\right\|_{0}^{2} d s \\
& +\mu_{*}\left\|t v_{m x}(t)\right\|_{0}^{2} \leq \frac{C_{T}}{\alpha_{*}} \int_{0}^{T}\left|\left(s^{2} \mu(s)\right)^{\prime}\right| d s \\
& +2 \int_{0}^{T}\left\|s \bar{f}_{1}(s)\right\|_{0}^{2} d s \\
& +2 T^{2}\left(\frac{R^{\gamma+1}-1}{\gamma+1}\right) \sup _{|z| \leq \bar{C}_{T}} f^{2}(z) d x \leq C_{T},
\end{aligned}
$$

for all $m \in \mathbb{N}$, for all $t \in[0, T]$, where $C_{T}$ always indicates a bound depending on $T$.

By $\left(t v_{m x}\right)^{\prime}=t v_{m x}^{\prime}+v_{m x}$ and (39) and (46), we deduce that

$$
\begin{aligned}
\left\|\left(t v_{m x}\right)^{\prime}\right\|_{L^{2}\left(Q_{T}\right)} \leq & \left\|t v_{m x}^{\prime}\right\|_{L^{2}\left(Q_{T}\right)}+\left\|v_{m x}\right\|_{L^{2}\left(Q_{T}\right)} \\
\leq & \sqrt{\int_{0}^{T}\left\|s v_{m x}^{\prime}(s)\right\|_{0}^{2} d s} \\
& +\sqrt{T}\left\|v_{m}\right\|_{L^{2}\left(0, T ; H_{0}^{1}\right)} \leq C_{T} .
\end{aligned}
$$

Step 3 (the limiting process). By (39), (46), and (47), we deduce that there exists a subsequence of $\left\{v_{m}\right\}$, still denoted by $\left\{v_{m}\right\}$ such that

$$
\begin{gathered}
v_{m} \longrightarrow v \quad \text { in } L^{\infty}\left(0, T ; H_{0}^{1}\right) \text { weakly }{ }^{*} \\
\left(t v_{m}\right)^{\prime} \longrightarrow(t v)^{\prime} \quad \text { in } L^{2}\left(0, T ; H_{0}^{1}\right) \text { weakly. }
\end{gathered}
$$

Using a compactness lemma ([18], Lions, p. 57), applied to (48), we can extract from the sequence $\left\{v_{m}\right\}$ a subsequence still denoted by $\left\{v_{m}\right\}$, such that

$$
t v_{m} \longrightarrow t v \text { strongly in } L^{2}\left(Q_{T}\right) \text {. }
$$

By the Riesz-Fischer theorem, we can extract from $\left\{v_{m}\right\}$ a subsequence still denoted by $\left\{v_{m}\right\}$, such that

$$
v_{m}(x, t) \longrightarrow v(x, t) \quad \text { a.e. }(x, t) \text { in } Q_{T} .
$$

Because $f$ is continuous, it gives

$$
\begin{array}{r}
f\left(v_{m}(x, t)+\varphi(x, t)\right) \rightarrow f(v(x, t)+\varphi(x, t)) \\
\text { a.e. }(x, t) \text { in } Q_{T} .
\end{array}
$$

On the other hand, by $\left(H_{6}\right)$, it follows from (44) that

$$
\left|f\left(v_{m}(x, t)+\varphi(x, t)\right)\right| \leq \sup _{|z| \leq \bar{C}_{T}}|f(z)| \leq C_{T},
$$

where $C_{T}$ is a constant independent of $m$. 
Using the dominated convergence theorem, (51) and (52) yield

$$
f\left(v_{m}+\varphi\right) \longrightarrow f(v+\varphi) \quad \text { strongly in } L^{2}\left(Q_{T}\right) .
$$

Passing to the limit in (30) by (31), (48), and (53), we obtain

$$
\begin{aligned}
& \frac{d}{d t}[\langle v(t), w\rangle+\alpha(t) a(v(t), w)] \\
& +\left(\mu(t)-\alpha^{\prime}(t)\right) a(v(t), w) \\
& +\langle f(v(t)+\varphi(t)), w\rangle=\left\langle\bar{f}_{1}(t), w\right\rangle, \\
& \quad \forall w \in H_{0}^{1}, \text { a.e., } t \in(0, T), \\
& v(0)=\widetilde{v}_{0} .
\end{aligned}
$$

Step 4 (uniqueness of the solution). First, we shall need the following lemma.

Lemma 7. Let $v$ be the weak solution of the following problem:

$$
\begin{aligned}
& v_{t}-\left(\mu(t)+\alpha(t) \frac{\partial}{\partial t}\right)\left(\frac{\partial^{2} v}{\partial x^{2}}+\frac{\gamma}{x} \frac{\partial v}{\partial x}\right)=\widetilde{f}(x, t), \\
& 1<x<R, 0<t<T, \\
& v(1, t)=v(R, t)=0, \\
& v(x, 0)=\widetilde{v}_{0}(x), \\
& v \in L^{\infty}\left(0, T ; H_{0}^{1}\right), t v_{t} \in L^{2}\left(0, T ; H_{0}^{1}\right), \mu, \alpha \in W^{1,1}(0, T) .
\end{aligned}
$$

Then,

$$
\begin{aligned}
& \|v(t)\|_{0}^{2}+\alpha(t)\left\|v_{x}(t)\right\|_{0}^{2} \\
& \quad+\int_{0}^{t}\left(2 \mu(s)-\alpha^{\prime}(s)\right)\left\|v_{x}(s)\right\|_{0}^{2} d s \\
& \geq\left\|\widetilde{v}_{0}\right\|_{0}^{2}+\alpha(0)\left\|\widetilde{v}_{0 x}\right\|_{0}^{2}+2 \int_{0}^{t}\langle\tilde{f}(s), v(s)\rangle d s .
\end{aligned}
$$

Furthermore, if $\widetilde{v}_{0}=0$, then the equality in (56) holds.

Lemma 7 is a slight improvement of a lemma used in [12] (or it can be found in Lions's book [18]).

Now, we will prove the uniqueness of the solutions.

Let $v_{1}$ and $v_{2}$ be two weak solutions of (24). Then, $v=v_{1}-$ $v_{2}$ is a weak solution of (55) with the right-hand side function replaced by $\tilde{f}(x, t)=-f\left(v_{1}+\varphi\right)+f\left(v_{2}+\varphi\right)$ and $\widetilde{v}_{0}=0$. Using Lemma 7 , we have equality

$$
\begin{aligned}
\sigma_{1}(t)= & -\int_{0}^{t}\left(2 \mu(s)-\alpha^{\prime}(s)\right)\left\|v_{x}(s)\right\|_{0}^{2} d s \\
& -2 \int_{0}^{t}\left\langle f\left(v_{1}+\varphi\right)-f\left(v_{2}+\varphi\right), v(s)\right\rangle d s,
\end{aligned}
$$

where

$$
\sigma_{1}(t)=\|v(t)\|_{0}^{2}+\alpha(t)\left\|v_{x}(t)\right\|_{0}^{2} .
$$

By $\left(H_{6}\right)$, we obtain

$$
\begin{gathered}
2 \int_{0}^{t}\left\langle f\left(v_{1}+\varphi\right)-f\left(v_{2}+\varphi\right), v(s)\right\rangle d s \\
\geq-2 \delta \int_{0}^{t}\|v(s)\|_{0}^{2} d s \geq-2 \delta \int_{0}^{t} \sigma_{1}(s) d s \\
-\int_{0}^{t}\left(2 \mu(s)-\alpha^{\prime}(s)\right)\left\|v_{x}(s)\right\|_{0}^{2} d s \\
\quad \leq \frac{1}{\alpha_{*}} \int_{0}^{t}\left|2 \mu(s)-\alpha^{\prime}(s)\right| \sigma_{1}(s) d s .
\end{gathered}
$$

It follows from (57)-(59) that

$$
\sigma_{1}(t) \leq \int_{0}^{t}\left(2 \delta+\frac{1}{\alpha_{*}}\left|2 \mu(s)-\alpha^{\prime}(s)\right|\right) \sigma_{1}(s) d s .
$$

By Gronwall's lemma, $v=0$.

Assume now that $\left(H_{5}\right)$ is replaced by $f_{1} \in L^{2}\left(Q_{T}\right)$; then we only have to show that $\left\{v_{m}^{\prime}\right\}$ is bounded in $L^{2}\left(0, T ; H_{0}^{1}\right)$.

Indeed, multiplying the $j$ th equation of (30) by $c_{m j}^{\prime}(t)$ and summing up with respect to $j$, afterwards, integrating with respect to the time variable from 0 to $t$, we get after some rearrangements

$$
\begin{aligned}
X_{m}(t)= & X_{m}(0)+\int_{0}^{t} \mu^{\prime}(s)\left\|v_{m x}(s)\right\|_{0}^{2} d s \\
& +2 \int_{0}^{t}\left\langle\bar{f}_{1}(s), v_{m}^{\prime}(s)\right\rangle d s \\
& -2 \int_{0}^{t}\left\langle f\left(v_{m}(s)+\varphi(s)\right), v_{m}^{\prime}(s)\right\rangle d s,
\end{aligned}
$$

where

$$
\begin{aligned}
X_{m}(t)= & 2 \int_{0}^{t}\left(\left\|v_{m}^{\prime}(s)\right\|_{0}^{2}+\alpha(s)\left\|v_{m x}^{\prime}(s)\right\|_{0}^{2}\right) d s \\
& +\mu(t)\left\|v_{m x}(t)\right\|_{0}^{2} .
\end{aligned}
$$

By the same estimates as above, we obtain

$$
\begin{aligned}
& X_{m}(0)=\mu(0)\left\|v_{0 m x}\right\|_{0}^{2} \leq \frac{\mu(0)}{\alpha_{*}} \bar{S}_{0} ; \\
& \int_{0}^{t} \mu^{\prime}(s)\left\|v_{m x}(s)\right\|_{0}^{2} d s \leq \int_{0}^{t} \frac{\left|\mu^{\prime}(s)\right|}{\alpha_{*}} S_{m}(s) d s \\
& \quad \leq \frac{C_{T}}{\alpha_{*}} \int_{0}^{T}\left|\mu^{\prime}(s)\right| d s \\
& 2 \int_{0}^{t}\left\langle\bar{f}_{1}(s), v_{m}^{\prime}(s)\right\rangle d s \\
& \quad \leq 2\left\|\bar{f}_{1}\right\|_{L^{2}\left(\mathrm{Q}_{T}\right)}^{2}+\frac{1}{2} \int_{0}^{t}\left\|v_{m}^{\prime}(s)\right\|_{0}^{2} d s \\
& \quad \leq 2\left\|\bar{f}_{1}\right\|_{L^{2}\left(\mathrm{Q}_{T}\right)}^{2}+\frac{1}{4} X_{m}(t) ;
\end{aligned}
$$




$$
\begin{aligned}
& -2 \int_{0}^{t}\left\langle f\left(v_{m}(s)+\varphi(s)\right), v_{m}^{\prime}(s)\right\rangle d s \\
& \leq 2 T\left(\frac{R^{\gamma+1}-1}{\gamma+1}\right) \sup _{|z| \leq \bar{C}_{T}} f^{2}(z)+\frac{1}{2} \int_{0}^{t}\left\|v_{m}^{\prime}(s)\right\|_{0}^{2} d s \\
& \quad \leq 2 T\left(\frac{R^{\gamma+1}-1}{\gamma+1}\right) \sup _{|z| \leq \bar{C}_{T}} f^{2}(z)+\frac{1}{4} X_{m}(t) .
\end{aligned}
$$

This implies

$$
\begin{aligned}
X_{m}(t) \leq & \frac{2}{\alpha_{*}}\left(\mu(0) \bar{S}_{0}+C_{T} \int_{0}^{T}\left|\mu^{\prime}(s)\right| d s\right) \\
& +4\left\|\bar{f}_{1}\right\|_{L^{2}\left(Q_{T}\right)}^{2} \\
& +4 T\left(\frac{R^{\gamma+1}-1}{\gamma+1}\right) \sup _{|z| \leq \bar{C}_{T}} f^{2}(z)=\bar{C}_{T}^{(1)} .
\end{aligned}
$$

Then, the sequence $\left\{v_{m}^{\prime}\right\}$ is bounded in $L^{2}\left(0, T ; H_{0}^{1}\right)$.

Applying a similar argument used as above, the limit $v$ of the sequence $\left\{v_{m}\right\}$ in suitable function spaces is a unique weak solution of (24) satisfying (28).

Therefore, Theorem 6 is proved.

\section{Asymptotic Behavior of the Solution}

as $t \rightarrow+\infty$

In this part, let $T>0,\left(H_{1}\right)-\left(H_{6}\right)$ hold. Then, there exists a unique solution $u=v+\varphi$ of problems (1)-(3) such that

$$
\begin{aligned}
u-\varphi & =v \in L^{\infty}\left(0, T ; H_{0}^{1}\right), \\
t\left(u_{t}-\varphi_{t}\right) & =t v_{t} \in L^{2}\left(0, T ; H_{0}^{1}\right) .
\end{aligned}
$$

We shall study asymptotic behavior of the solution $u(t)$ as $t \rightarrow+\infty$.

We make the following supplementary assumptions on the functions $g_{1}(t), g_{R}(t), \alpha(t), \mu(t)$, and $f_{1}(x, t)$ :

$\left(H_{2}^{\prime}\right) g_{1}, g_{R} \in W^{1,1}\left(\mathbb{R}_{+}\right), \widetilde{u}_{0}(1)-g_{1}(0)=\widetilde{u}_{0}(R)-g_{R}(0)=0$, and there exist the positive constants $\bar{C}_{1}, \bar{C}_{R}, \bar{\gamma}_{1}$, and $\bar{\gamma}_{R}$, such that

$$
\left|g_{i}(t)\right|+\left|g_{i}^{\prime}(t)\right| \leq \bar{C}_{i} e^{-\bar{\gamma}_{i} t}, \quad \forall t \geq 0, i \in\{1, R\} .
$$

$\left(H_{3}^{\prime}\right) \alpha \in W^{1,1}\left(\mathbb{R}_{+}\right), \alpha(t) \geq \alpha_{*}>0, \forall t \geq 0, \alpha(0) \leq \alpha(T)$.

$\left(H_{4}^{\prime}\right) \mu \in W^{1,1}\left(\mathbb{R}_{+}\right)$, and there exist the positive constants $\mu_{*}, \bar{\mu}_{*}, \mu_{\infty}, \bar{C}_{\mu}$, and $\bar{\gamma}_{\mu}$, such that

$$
\begin{aligned}
& \mu(t) \geq \mu_{*}>0, \forall t \geq 0, \\
&\left|\mu(t)-\mu_{\infty}\right| \leq \bar{C}_{\mu} e^{-\bar{\gamma}_{\mu} t}, \quad \forall t \geq 0, \\
& 2 \mu(t)-\alpha^{\prime}(t) \geq 2 \bar{\mu}_{*}>0, \quad \forall t \geq 0 .
\end{aligned}
$$

$\left(H_{5}^{\prime}\right) f_{1} \in L^{\infty}\left(0, \infty ; L^{2}\right)$, and there exist the positive constants $C_{1}, \gamma_{1}$ and the function $f_{1 \infty} \in L^{2}$, such that $\left\|f_{1}(t)-f_{1 \infty}\right\|_{0} \leq C_{1} e^{-\gamma_{1} t}, \forall t \geq 0$.

$\left(H_{6}^{\prime}\right) f \in C^{0}(\mathbb{R} ; \mathbb{R})$ and there exists a positive constant $\delta$, with $0<\delta<2 \mu_{\infty} / R^{\gamma}(R-1)^{2}$, such that $(y-z)(f(y)-$ $f(z)) \geq-\delta|y-z|^{2}$, for all $y, z \in \mathbb{R}$.

First, we consider the following stationary problem:

$$
\begin{aligned}
&-\mu_{\infty}\left(\frac{\partial^{2} u}{\partial x^{2}}+\frac{\gamma}{x} \frac{\partial u}{\partial x}\right)+f(u)=f_{1 \infty}(x), \\
& 1<x<R, \\
& u(1, t)=u(R, t)=0 .
\end{aligned}
$$

The weak solution of problem (68) is obtained from the following variational problem. Find $u_{\infty} \in H_{0}^{1}$ such that

$$
\mu_{\infty} a\left(u_{\infty}, w\right)+\left\langle f\left(u_{\infty}\right), w\right\rangle=\left\langle f_{1 \infty}, w\right\rangle
$$

for all $w \in H_{0}^{1}$, where $a(\cdot, \cdot)$ is the symmetric bilinear form on $H_{0}^{1} \times H_{0}^{1}$ defined by (21).

We then have the following theorem.

Theorem 8. Let $\left(H_{4}^{\prime}\right)-\left(H_{6}^{\prime}\right)$ hold. Then, there exists a unique solution $u_{\infty}$ of the variational problem (69) such that $u_{\infty} \in H_{0}^{1}$.

Proof. Consider the basis $\left\{w_{j}\right\}$ for $H_{0}^{1}$ as in Lemma 5. Put

$$
y_{m}=\sum_{j=1}^{m} d_{m j} w_{j}
$$

where $d_{m j}$ satisfies the following nonlinear equation system:

$$
\begin{aligned}
\mu_{\infty} a\left(y_{m}, w_{j}\right)+\left\langle f\left(y_{m}\right), w_{j}\right\rangle=\left\langle f_{1 \infty}, w_{j}\right\rangle & \\
& 1 \leq j \leq m .
\end{aligned}
$$

By Brouwer's lemma (see Lions [18], Lemma 4.3, p. 53), it follows from the hypotheses $\left(H_{4}^{\prime}\right)-\left(H_{6}^{\prime}\right)$ that systems $(70)$ and (71) have a solution $y_{m}$.

Multiplying the $j$ th equation of system (71) by $d_{m j}$, and then summing up with respect to $j$, we have

$$
\mu_{\infty} a\left(y_{m}, y_{m}\right)+\left\langle f\left(y_{m}\right), y_{m}\right\rangle=\left\langle f_{1 \infty}, y_{m}\right\rangle
$$

By using $\left(H_{6}\right)$, we obtain

$$
\begin{aligned}
\left\langle f\left(y_{m}\right), y_{m}\right\rangle= & \int_{1}^{R} x^{\gamma}\left(f\left(y_{m}(x)\right)-f(0)\right) y_{m}(x) d x \\
& +\int_{1}^{R} x^{\gamma} f(0) y_{m}(x) d x
\end{aligned}
$$




$$
\begin{aligned}
\geq & -\delta \int_{1}^{R} x^{\gamma} y_{m}^{2}(x) d x \\
& +\int_{1}^{R} x^{\gamma} f(0) y_{m}(x) d x \\
\geq & -\delta\left\|y_{m}\right\|_{0}^{2}-\varepsilon_{1}\left\|y_{m}\right\|_{0}^{2} \\
& -\frac{1}{4 \varepsilon_{1}} \int_{1}^{R} x^{\gamma} f^{2}(0) d x \\
= & -\left(\delta+\varepsilon_{1}\right)\left\|y_{m}\right\|_{0}^{2} \\
& -\frac{1}{4 \varepsilon_{1}}\left(\frac{R^{\gamma+1}-1}{\gamma+1}\right) f^{2}(0),
\end{aligned}
$$$$
\forall \varepsilon_{1}>0 \text {. }
$$

By using inequalities (20)(iii) and (73), we obtain from (72) that

$$
\begin{aligned}
\mu_{\infty}\left\|y_{m x}\right\|_{0}^{2} \leq & \left(\delta+\varepsilon_{1}\right)\left\|y_{m}\right\|_{0}^{2} \\
& +\frac{1}{4 \varepsilon_{1}}\left(\frac{R^{\gamma+1}-1}{\gamma+1}\right) f^{2}(0) \\
& +\left\|f_{1 \infty}\right\|_{0}\left\|y_{m}\right\|_{0} \\
\leq & \left(\delta+\varepsilon_{1}\right)\left\|y_{m}\right\|_{0}^{2} \\
& +\frac{1}{4 \varepsilon_{1}}\left(\frac{R^{\gamma+1}-1}{\gamma+1}\right) f^{2}(0) \\
& +\frac{1}{4 \varepsilon_{1}}\left\|f_{1 \infty}\right\|_{0}^{2}+\varepsilon_{1}\left\|y_{m}\right\|_{0}^{2} \\
= & \left(\delta+2 \varepsilon_{1}\right)\left\|y_{m}\right\|_{0}^{2} \\
& +\frac{1}{4 \varepsilon_{1}}\left[\left(\frac{R^{\gamma+1}-1}{\gamma+1}\right) f^{2}(0)+\left\|f_{1 \infty}\right\|_{0}^{2}\right] \\
= & \left(\delta+2 \varepsilon_{1}\right) \frac{R^{\gamma}}{2}(R-1)^{2}\left\|y_{m x}\right\|_{0}^{2} \\
& +\frac{1}{4 \varepsilon_{1}}\left[\left(\frac{R^{\gamma+1}-1}{\gamma+1}\right) f^{2}(0)+\left\|f_{1 \infty}\right\|_{0}^{2}\right] .
\end{aligned}
$$

By $0<\delta<2 \mu_{\infty} / R^{\gamma}(R-1)^{2}$, choose $\varepsilon_{1}>0$ such that $\left(\delta+2 \varepsilon_{1}\right)\left(R^{\gamma} / 2\right)(R-1)^{2}<\mu_{\infty}$.

Hence, we deduce from (74) that

$$
\begin{aligned}
\left\|y_{m x}\right\|_{0} & \leq \sqrt{\frac{\left(\left(R^{\gamma+1}-1\right) /(\gamma+1)\right) f^{2}(0)+\left\|f_{1 \infty}\right\|_{0}^{2}}{4 \varepsilon_{1}\left[\mu_{\infty}-\left(\delta+2 \varepsilon_{1}\right)\left(R^{\gamma} / 2\right)(R-1)^{2}\right]}} \\
& =\widetilde{D}_{1},
\end{aligned}
$$

and $\widetilde{D}_{1}$ is a constant independent of $m$.
By means of (75) and Lemma 3, the sequence $\left\{y_{m}\right\}$ has a subsequence still denoted by $\left\{y_{m}\right\}$ such that

$$
\begin{aligned}
& y_{m} \longrightarrow u_{\infty} \quad \text { in } H_{0}^{1} \text { weakly, } \\
& y_{m} \longrightarrow u_{\infty} \quad \text { in } C^{0}([1, R]) \text { strongly. }
\end{aligned}
$$
have

On the other hand, by $(76)_{2}$ and the continuity of $f$, we

$$
f\left(y_{m}\right) \longrightarrow f\left(u_{\infty}\right) \text { in } C^{0}([1, R]) \text { strongly. }
$$

Passing to the limit in (71), we find without difficulty from (76) and (77) that $u_{\infty}$ satisfies the equation

$$
\mu_{\infty} a\left(u_{\infty}, w_{j}\right)+\left\langle f\left(u_{\infty}\right), w_{j}\right\rangle=\left\langle f_{1 \infty}, w_{j}\right\rangle .
$$

Equation (78) holds for every $j=1,2, \ldots$; that is, (69) is true.

The solution of problem (69) is unique, which can be shown by the same arguments as in the proof of Theorem 6.

Now we consider asymptotic behavior of the solution $u(t)$ as $t \rightarrow+\infty$.

We then have the following theorem.

Theorem 9. Let $\left(H_{1}\right),\left(H_{2}^{\prime}\right)-\left(H_{5}^{\prime}\right)$, and $\left(H_{6}\right)$ hold. Let $f$ satisfy the following condition, in addition,

$$
\begin{aligned}
& \left(H_{6}^{\prime \prime}\right) \forall M>0, \exists k_{M}>0:|f(y)-f(z)| \leq k_{M}|y-z| \text {, } \\
& \forall y, z \in[-M, M] \text {. }
\end{aligned}
$$

And let $\delta>0$ in $\left(\mathrm{H}_{6}\right)$ satisfy the following condition, in addition,

$$
\left(H_{6}^{\prime \prime \prime}\right) 0<\delta<\left(2 / R^{\gamma}(R-1)^{2}\right) \min \left\{\mu_{\infty}, \bar{\mu}_{*}\right\} .
$$

Then we have

$$
\left\|u(t)-u_{\infty}\right\|_{1} \leq \bar{C} e^{-\bar{\gamma} t}, \quad \forall t \geq 0
$$

where $\bar{\gamma}>0, \bar{C}>0$ are constants independent of $t$.

Proof. Put $Z_{m}(t)=v_{m}(t)-y_{m}$. Let us subtract (30) 1 with (71) to obtain

$$
\begin{aligned}
& \left\langle Z_{m}^{\prime}(t), w_{j}\right\rangle+\alpha(t) a\left(Z_{m}^{\prime}(t), w_{j}\right) \\
& \quad+\mu(t) a\left(Z_{m}(t), w_{j}\right)+\left(\mu(t)-\mu_{\infty}\right) a\left(y_{m}, w_{j}\right) \\
& +\left\langle f\left(v_{m}(t)+\varphi(t)\right)-f\left(y_{m}\right), w_{j}\right\rangle \\
& \quad=\left\langle\bar{f}_{1}(t)-f_{1 \infty}, w_{j}\right\rangle, \quad 1 \leq j \leq m, \\
& Z_{m}(0)=v_{0 m}-y_{m} .
\end{aligned}
$$


By multiplying (80) $)_{1}$ by $c_{m j}(t)-d_{m j}$ and summing up in $j$, we obtain

$$
\begin{aligned}
\frac{d}{d t}[ & \left.\left\|Z_{m}(t)\right\|_{0}^{2}+\alpha(t)\left\|Z_{m x}(t)\right\|_{0}^{2}\right] \\
& +\left(2 \mu(t)-\alpha^{\prime}(t)\right)\left\|Z_{m x}(t)\right\|_{0}^{2} \\
& +2\left(\mu(t)-\mu_{\infty}\right) a\left(y_{m}, Z_{m}(t)\right) \\
& +2\left\langle f\left(v_{m}(t)+\varphi(t)\right)-f\left(y_{m}+\varphi(t)\right), Z_{m}(t)\right\rangle \\
& +2\left\langle f\left(y_{m}+\varphi(t)\right)-f\left(y_{m}\right), Z_{m}(t)\right\rangle \\
= & 2\left\langle\bar{f}_{1}(t)-f_{1}(t), Z_{m}(t)\right\rangle \\
& +2\left\langle f_{1}(t)-f_{1 \infty}, Z_{m}(t)\right\rangle .
\end{aligned}
$$

By the assumptions $\left(H_{2}^{\prime}\right)-\left(H_{5}^{\prime}\right),\left(H_{6}\right),\left(H_{6}^{\prime \prime}\right)$, and $\left(H_{6}^{\prime \prime \prime}\right)$ and using inequality (20)(iii), and with $\varepsilon_{1}>0$, we estimate without difficulty the following terms in (81) as follows:

(i) Estimate $\left(2 \mu(t)-\alpha^{\prime}(t)\right)\left\|Z_{m x}(t)\right\|_{0}^{2}$, as

$$
\left(2 \mu(t)-\alpha^{\prime}(t)\right)\left\|Z_{m x}(t)\right\|_{0}^{2} \geq 2 \bar{\mu}_{*}\left\|Z_{m x}(t)\right\|_{0}^{2} ;
$$

(ii) Estimate $2\left(\mu(t)-\mu_{\infty}\right) a\left(y_{m}, Z_{m}(t)\right)$, as

$$
\begin{aligned}
& 2\left(\mu(t)-\mu_{\infty}\right) a\left(y_{m}, Z_{m}(t)\right) \\
& \quad \leq 2\left|\mu(t)-\mu_{\infty}\right|\left\|y_{m x}\right\|_{0}\left\|Z_{m x}(t)\right\|_{0} \\
& \quad \leq 2 \bar{C}_{\mu} e^{-\bar{\gamma}_{\mu} t} \widetilde{C}\left\|Z_{m x}(t)\right\|_{0} \\
& \quad \leq \frac{1}{\varepsilon_{1}} \widetilde{C}^{2} \bar{C}_{\mu}^{2} e^{-2 \bar{\gamma}_{\mu} t}+\varepsilon_{1}\left\|Z_{m x}(t)\right\|_{0}^{2} .
\end{aligned}
$$

(iii) Estimate $2\left\langle f\left(v_{m}(t)+\varphi(t)\right)-f\left(y_{m}+\varphi(t)\right), Z_{m}(t)\right\rangle$, as

$$
\begin{aligned}
& 2\left\langle f\left(v_{m}(t)+\varphi(t)\right)-f\left(y_{m}+\varphi(t)\right), Z_{m}(t)\right\rangle \\
& \quad \geq-2 \delta\left\|Z_{m}(t)\right\|_{0}^{2} \geq-2 \delta \frac{R^{\gamma}}{2}(R-1)^{2}\left\|Z_{m x}(t)\right\|_{0}^{2} .
\end{aligned}
$$

(iv) Estimate $2\left\langle f\left(y_{m}+\varphi(t)\right)-f\left(y_{m}\right), Z_{m}(t)\right\rangle$. Note that, from the inequalities

$$
\begin{array}{r}
|\varphi(x, t)| \leq\left|g_{1}(t)\right|+\left|g_{R}(t)\right| \leq \bar{C}_{1}+\bar{C}_{R}, \\
\left\|y_{m}\right\|_{C^{0}(\bar{\Omega})} \leq \sqrt{R-1}\left\|y_{m x}\right\| \leq \sqrt{R-1} \widetilde{D}_{1}, \\
\left\|y_{m}+\varphi\right\|_{C^{0}(\bar{\Omega})} \leq \sqrt{R-1} \widetilde{D}_{1}+\bar{C}_{1}+\bar{C}_{R}=M_{1},
\end{array}
$$

and $\left(H_{6}^{\prime \prime}\right)$, we deduce that

$$
\begin{gathered}
\left|f\left(y_{m}+\varphi(t)\right)-f\left(y_{m}\right)\right| \leq k_{M_{1}}|\varphi(x, t)| \\
\leq k_{M_{1}}\left(\left|g_{1}(t)\right|+\left|g_{R}(t)\right|\right)=k_{M_{1}} \Psi(t),
\end{gathered}
$$

where

$$
\Psi(t)=\bar{C}_{1} e^{-\bar{\gamma}_{1} t}+\bar{C}_{R} e^{-\bar{\gamma}_{R} t}
$$

Hence,

$$
\begin{aligned}
& \left\|f\left(y_{m}+\varphi(t)\right)-f\left(y_{m}\right)\right\|_{0}^{2} \\
& \quad \leq \frac{1}{\gamma+1}\left(R^{\gamma+1}-1\right) k_{M_{1}}^{2} \Psi^{2}(t) .
\end{aligned}
$$

Thus,

$$
\begin{aligned}
& 2\left\langle f\left(y_{m}+\varphi(t)\right)-f\left(y_{m}\right), Z_{m}(t)\right\rangle \\
& \leq \frac{1}{\varepsilon_{1}}\left\|f\left(y_{m}+\varphi(t)\right)-f\left(y_{m}\right)\right\|_{0}^{2}+\varepsilon_{1}\left\|Z_{m}(t)\right\|_{0}^{2} \\
& \leq \frac{1}{\varepsilon_{1}} \frac{1}{\gamma+1}\left(R^{\gamma+1}-1\right) k_{M_{1}}^{2} \Psi^{2}(t) \\
&+\varepsilon_{1} \frac{R^{\gamma}}{2}(R-1)^{2}\left\|Z_{m x}(t)\right\|_{0}^{2} .
\end{aligned}
$$

(v) Estimate $\left\langle\bar{f}_{1}(t)-f_{1}(t), Z_{m}(t)\right\rangle$. We have

$$
\begin{aligned}
& \bar{f}_{1}(x, t)-f_{1}(x, t)=-\frac{1}{R-1}\left[(x-1) g_{R}^{\prime}(t)\right. \\
& \left.+(R-x) g_{1}^{\prime}(t)\right] \\
& +\frac{\gamma}{(R-1) x}\left[\mu(t)\left(g_{R}(t)-g_{1}(t)\right)\right. \\
& \left.+\alpha(t)\left(g_{R}^{\prime}(t)-g_{1}^{\prime}(t)\right)\right] .
\end{aligned}
$$

Hence,

$$
\begin{aligned}
& \left|\bar{f}_{1}(x, t)-f_{1}(x, t)\right| \leq\left|g_{1}^{\prime}(t)\right|+\left|g_{R}^{\prime}(t)\right| \\
& +\frac{\gamma}{R-1}(\alpha(t)+\mu(t)) \\
& \cdot\left[\left|g_{1}(t)\right|+\left|g_{1}^{\prime}(t)\right|+\left|g_{R}(t)\right|+\left|g_{R}^{\prime}(t)\right|\right] \leq \bar{C}_{1} e^{-\bar{\gamma}_{1} t} \\
& +\bar{C}_{R} e^{-\bar{\gamma}_{R} t}+\frac{\gamma}{R-1}\left(\|\alpha\|_{L^{\infty}\left(\mathbb{R}_{+}\right)}+\|\mu\|_{L^{\infty}\left(\mathbb{R}_{+}\right)}\right) \\
& \cdot\left[\bar{C}_{1} e^{-\bar{\gamma}_{1} t}+\bar{C}_{R} e^{-\bar{\gamma}_{R^{t}}}\right] \\
& =\left[1+\frac{\gamma}{R-1}\left(\|\alpha\|_{L^{\infty}\left(\mathbb{R}_{+}\right)}+\|\mu\|_{L^{\infty}\left(\mathbb{R}_{+}\right)}\right)\right] \\
& \cdot\left[\bar{C}_{1} e^{-\bar{\gamma}_{1} t}+\bar{C}_{R} e^{-\bar{\gamma}_{R^{t}} t}\right] \\
& =\left[1+\frac{\gamma}{R-1}\left(\|\alpha\|_{L^{\infty}\left(\mathbb{R}_{+}\right)}+\|\mu\|_{L^{\infty}\left(\mathbb{R}_{+}\right)}\right)\right] \Psi(t) \\
& \equiv \widetilde{C}(\alpha, \mu) \Psi(t) .
\end{aligned}
$$

It follows that

$\left\|\bar{f}_{1}(t)-f_{1}(t)\right\|_{0}^{2} \leq \frac{1}{\gamma+1}\left(R^{\gamma+1}-1\right) \widetilde{C}^{2}(\alpha, \mu) \Psi^{2}(t)$. 
Thus,

$$
\begin{aligned}
& 2\left\langle\bar{f}_{1}(t)-f_{1}(t), Z_{m}(t)\right\rangle \\
& \leq \frac{1}{\varepsilon_{1}}\left\|\bar{f}_{1}(t)-f_{1}(t)\right\|_{0}^{2}+\varepsilon_{1}\left\|Z_{m}(t)\right\|_{0}^{2} \\
& \leq \frac{1}{\varepsilon_{1}} \frac{1}{\gamma+1}\left(R^{\gamma+1}-1\right) \widetilde{C}^{2}(\alpha, \mu) \Psi^{2}(t) \\
&+\varepsilon_{1} \frac{R^{\gamma}}{2}(R-1)^{2}\left\|Z_{m x}(t)\right\|_{0}^{2} .
\end{aligned}
$$

(vi) Estimate $\left\langle f_{1}(t)-f_{1 \infty}, Z_{m}(t)\right\rangle$, as

$$
\begin{aligned}
& 2\left\langle f_{1}(t)-f_{1 \infty}, Z_{m}(t)\right\rangle \\
& \quad \leq \frac{1}{\varepsilon_{1}}\left\|f_{1}(t)-f_{1 \infty}\right\|_{0}^{2}+\varepsilon_{1}\left\|Z_{m}(t)\right\|_{0}^{2} \\
& \quad \leq \frac{1}{\varepsilon_{1}} C_{1}^{2} e^{-2 \gamma_{1} t}+\varepsilon_{1} \frac{R^{\gamma}}{2}(R-1)^{2}\left\|Z_{m x}(t)\right\|_{0}^{2} .
\end{aligned}
$$

It follows from (81)-(84), (89), (93), and (94) that

$$
\begin{aligned}
\frac{d}{d t} & {\left[\left\|Z_{m}(t)\right\|_{0}^{2}+\alpha(t)\left\|Z_{m x}(t)\right\|_{0}^{2}\right] } \\
& +\left[2 \bar{\mu}_{*}-\varepsilon_{1}-\left(2 \delta+3 \varepsilon_{1}\right) \frac{R^{\gamma}}{2}(R-1)^{2}\right]\left\|Z_{m x}(t)\right\|_{0}^{2} \\
& \leq \frac{1}{\varepsilon_{1}}\left(\widetilde{C}^{2} \bar{C}_{\mu}^{2} e^{-2 \bar{\gamma}_{\mu} t}+C_{1}^{2} e^{-2 \gamma_{1} t}\right) \\
& +\frac{1}{\varepsilon_{1}} \frac{R^{\gamma+1}-1}{\gamma+1}\left(k_{M_{1}}^{2}+\widetilde{C}^{2}(\alpha, \mu)\right) \Psi^{2}(t) \equiv \widetilde{\psi}(t) .
\end{aligned}
$$

By $0<\delta<\left(2 / R^{\gamma}(R-1)^{2}\right) \min \left\{\mu_{\infty}, \bar{\mu}_{*}\right\} \leq 2 \bar{\mu}_{*} / R^{\gamma}(R-1)^{2}$, choose $\varepsilon_{1}>0$ such that $2 \widetilde{\gamma}=2 \bar{\mu}_{*}-\varepsilon_{1}-\left(2 \delta+3 \varepsilon_{1}\right)\left(R^{\gamma} / 2\right)(R-$ $1)^{2}>0$.

Put $\bar{\gamma}_{0}=\min \left\{\gamma_{1}, \bar{\gamma}_{1}, \bar{\gamma}_{R}, \bar{\gamma}_{\mu}\right\}$, and we have $\widetilde{\psi}(t) \leq \bar{C}_{0} e^{-2 \bar{\gamma}_{0} t}$ for all $t \geq 0$, as

$$
\begin{aligned}
& \frac{d}{d t}\left[\left\|Z_{m}(t)\right\|_{0}^{2}+\alpha(t)\left\|Z_{m x}(t)\right\|_{0}^{2}\right]+2 \widetilde{\gamma}\left\|Z_{m x}(t)\right\|_{0}^{2} \\
& \quad \leq \widetilde{\psi}(t) \leq \bar{C}_{0} e^{-2 \bar{\gamma}_{0} t} .
\end{aligned}
$$

By

$$
\begin{aligned}
\left\|Z_{m x}(t)\right\|_{0}^{2}= & \frac{1}{2}\left\|Z_{m x}(t)\right\|_{0}^{2}+\frac{1}{2}\left\|Z_{m x}(t)\right\|_{0}^{2} \\
\geq & \frac{1}{2} \frac{1}{\alpha(t)} \alpha(t)\left\|Z_{m x}(t)\right\|_{0}^{2} \\
& +\frac{1}{2} \frac{2}{R^{\gamma}(R-1)^{2}}\left\|Z_{m}(t)\right\|_{0}^{2}
\end{aligned}
$$

$$
\begin{aligned}
\geq & \frac{1}{2} \frac{1}{\|\alpha\|_{L^{\infty}\left(\mathbb{R}_{+}\right)}} \alpha(t)\left\|Z_{m x}(t)\right\|_{0}^{2} \\
& +\frac{1}{2} \frac{2}{R^{\gamma}(R-1)^{2}}\left\|Z_{m}(t)\right\|_{0}^{2} \\
\geq & \beta_{1}\left(\alpha(t)\left\|Z_{m x}(t)\right\|_{0}^{2}+\left\|Z_{m}(t)\right\|_{0}^{2}\right),
\end{aligned}
$$

where $\beta_{1}=(1 / 2) \min \left\{1 /\|\alpha\|_{L^{\infty}\left(\mathbb{R}_{+}\right)}, 2 / R^{\gamma}(R-1)^{2}\right\}$, it follows from (96) and (97) that

$$
\begin{aligned}
& \frac{d}{d t}\left[\left\|Z_{m}(t)\right\|_{0}^{2}+\alpha(t)\left\|Z_{m x}(t)\right\|_{0}^{2}\right] \\
& +2 \widetilde{\gamma} \beta_{1}\left(\left\|Z_{m}(t)\right\|_{0}^{2}+\alpha(t)\left\|Z_{m x}(t)\right\|_{0}^{2}\right) \leq \widetilde{\psi}(t) \\
& \quad \leq \bar{C}_{0} e^{-2 \bar{\gamma}_{0} t} .
\end{aligned}
$$

Choose $\bar{\gamma}>0$ such that $\bar{\gamma}<\min \left\{\bar{\gamma}_{0}, 2 \widetilde{\gamma} \beta_{1}\right\}$, and then we have from (98) that

$$
\begin{aligned}
& \frac{d}{d t}\left[\left\|Z_{m}(t)\right\|_{0}^{2}+\alpha(t)\left\|Z_{m x}(t)\right\|_{0}^{2}\right] \\
& \quad+2 \bar{\gamma}\left(\left\|Z_{m}(t)\right\|_{0}^{2}+\alpha(t)\left\|Z_{m x}(t)\right\|_{0}^{2}\right) \leq \bar{C}_{0} e^{-2 \bar{\gamma}_{0} t} .
\end{aligned}
$$

Hence, we obtain from (99) that

$$
\begin{aligned}
& \left\|Z_{m}(t)\right\|_{0}^{2}+\alpha_{*}\left\|Z_{m x}(t)\right\|_{0}^{2} \leq\left\|Z_{m}(t)\right\|_{0}^{2}+\alpha(t) \\
& \cdot\left\|Z_{m x}(t)\right\|_{0}^{2} \\
& \leq\left(\left\|Z_{m}(0)\right\|_{0}^{2}+\alpha(0)\left\|Z_{m x}(0)\right\|_{0}^{2}+\frac{\bar{C}_{0}}{2\left(\bar{\gamma}_{0}-\bar{\gamma}\right)}\right) \\
& \cdot e^{-2 \bar{\gamma} t} .
\end{aligned}
$$

Letting $m \rightarrow+\infty$ in (100), we obtain

$$
\begin{aligned}
& \left\|v(t)-u_{\infty}\right\|_{0}^{2}+\alpha_{*}\left\|v_{x}(t)-u_{\infty x}\right\|_{0}^{2} \\
& \quad \leq \liminf _{m \rightarrow+\infty}\left(\left\|v_{m}(t)-y_{m}\right\|_{0}^{2}+\alpha_{*}\left\|v_{m x}(t)-y_{m x}\right\|_{0}^{2}\right) \\
& \leq\left(\left\|\widetilde{v}_{0}-u_{\infty}\right\|_{0}^{2}+\alpha(0)\left\|\widetilde{v}_{0 x}-u_{\infty x}\right\|_{0}^{2}\right. \\
& \left.\quad+\frac{\bar{C}_{0}}{2\left(\bar{\gamma}_{0}-\bar{\gamma}\right)}\right) e^{-2 \bar{\gamma} t}, \quad \forall t \geq 0,
\end{aligned}
$$

or

$$
\left\|v(t)-u_{\infty}\right\|_{1} \leq \widetilde{D}_{2} e^{-\bar{\gamma} t}, \quad \forall t \geq 0,
$$

where

$$
\widetilde{D}_{2}=\sqrt{\frac{1}{\min \left(1, \alpha_{*}\right)}\left(\left\|\widetilde{v}_{0}-u_{\infty}\right\|_{0}^{2}+\alpha(0)\left\|\widetilde{v}_{0 x}-u_{\infty x}\right\|_{0}^{2}+\frac{\bar{C}_{0}}{2\left(\bar{\gamma}_{0}-\bar{\gamma}\right)}\right)} .
$$


Note that

$$
\begin{aligned}
|\varphi(x, t)| & \leq\left|g_{R}(t)\right|+\left|g_{1}(t)\right| \leq \bar{C}_{1} e^{-\bar{\gamma}_{1} t}+\bar{C}_{R} e^{-\bar{\gamma}_{R} t} \\
& =\Psi(t) ; \\
\left|\varphi_{x}(x, t)\right| & =\frac{\left|g_{R}(t)-g_{1}(t)\right|}{R-1} \leq \frac{1}{R-1} \Psi(t) .
\end{aligned}
$$

Hence,

$$
\begin{aligned}
\|\varphi(t)\|_{1}^{2} & =\|\varphi(t)\|_{0}^{2}+\left\|\varphi_{x}(t)\right\|_{0}^{2} \\
& \leq \frac{1}{\gamma+1}\left(R^{\gamma+1}-1\right)\left(1+\frac{1}{(R-1)^{2}}\right) \Psi^{2}(t) \\
& \leq \widetilde{D}_{3}^{2} e^{-2 \bar{\gamma} t} .
\end{aligned}
$$

It follows from (102) and (105) that

$$
\begin{aligned}
\left\|u(t)-u_{\infty}\right\|_{1} & \leq\left\|v(t)-u_{\infty}\right\|_{1}+\|\varphi(t)\|_{1} \\
& \leq\left(\widetilde{D}_{2}+\widetilde{D}_{3}\right) e^{-\bar{\gamma} t}, \quad \forall t \geq 0 .
\end{aligned}
$$

This completes the proof of Theorem 9.

\section{The Existence and the Uniqueness of a $(\eta, T)$-Periodic Weak Solution}

In this section, we shall consider problems (1), (2), and (4) with $R>1,0<|\eta| \leq 1$ as given constants and $\mu, \alpha$, $f, f_{1}, g_{1}$, and $g_{R}$ as given functions satisfying the following assumptions:

$\left(\bar{H}_{2}\right) g_{1}, g_{R} \in W^{1,1}(0, T)$, and $g_{1}$ and $g_{R}$ are $(\eta, T)$-periodic; that is,

$$
\begin{aligned}
& g_{1}(0)=\eta g_{1}(T), \\
& g_{R}(0)=\eta g_{R}(T) .
\end{aligned}
$$

$\left(\bar{H}_{3}\right) \alpha \in W^{1,1}(0, T), \alpha(t) \geq \alpha_{*}>0, \forall t \in[0, T], 0<\alpha(0) \leq$ $\alpha(T)$;

$\left(\bar{H}_{4}\right) \mu \in W^{1,1}(0, T), \mu(t) \geq \mu_{*}>0, \forall t \in[0, T], 2 \mu(t)-$ $\alpha^{\prime}(t) \geq 2 \bar{\mu}_{*}>0$, a.e., $t \in[0, T]$

$\left(\bar{H}_{5}\right) f_{1}, f_{1}^{\prime} \in L^{2}\left(Q_{T}\right), f_{1}$ is $(\eta, T)$-periodic, $f_{1}(0)=\eta f_{1}(T)$;

$\left(\bar{H}_{6}\right) f \in C^{0}(\mathbb{R} ; \mathbb{R})$ and there exists a positive constant $\delta$, with

$$
0<\delta<\frac{2 \bar{\mu}_{*}}{R^{\gamma}(R-1)^{2}},
$$

such that $(y-z)(f(y)-f(z)) \geq-\delta|y-z|^{2}$,

$$
\forall y, z \in \mathbb{R} .
$$

Remark 10. An example of the functions $g_{1}, g_{R}$ satisfying $\left(\bar{H}_{2}\right)$ is

$$
g_{k}(t)=\zeta_{k} e^{p t},
$$

where $p>0, \zeta_{k}$, and $k \in\{1, R\}$ are constants. It is obvious that $\left(\bar{H}_{2}\right)$ holds, because

$$
g_{k}(t)=e^{-p T} \zeta_{k} e^{p(t+T)}=\eta g_{k}(t+T),
$$

with $\eta=e^{-p T}$ and

$$
g_{k}(0)=\eta g_{k}(T), \quad k \in\{1, R\} .
$$

Similarly, by the transformation $v(x, t)=u(x, t)-\varphi(x, t)$, with $\varphi(x, t)=((x-1) /(R-1)) g_{R}(t)+((R-x) /(R-1)) g_{1}(t)$, $\varphi(x, 0)=\eta \varphi(x, T)$, problems (1), (2), and (4) reduce to the following problem:

$$
\begin{aligned}
& v_{t}-\left(\mu(t)+\alpha(t) \frac{\partial}{\partial t}\right)\left(\frac{\partial^{2} v}{\partial x^{2}}+\frac{\gamma}{x} \frac{\partial v}{\partial x}\right)+f(v+\varphi) \\
& \quad=\bar{f}_{1}(x, t), \quad 1<x<R, 0<t<T, \\
& v(1, t)=v(R, t)=0, \\
& v(x, 0)=\eta v(x, T),
\end{aligned}
$$

where $\bar{f}_{1}(x, t)$ is defined by $(25)_{1}$.

The weak formulation of problem (112) can be given in the following manner: Find $v \in L^{\infty}\left(0, T ; H_{0}^{1}\right)$ with $v_{t} \in$ $L^{2}\left(0, T ; H_{0}^{1}\right)$, such that $v$ satisfies the following variational equation:

$$
\begin{aligned}
& \int_{0}^{T}\left\langle v^{\prime}(t), w(t)\right\rangle d t+\int_{0}^{T} \alpha(t) a\left(v^{\prime}(t), w(t)\right) d t \\
& \quad+\int_{0}^{T} \mu(t) a(v(t), w(t)) d t \\
& \quad+\int_{0}^{T}\langle f(v(t)+\varphi(t)), w(t)\rangle d t \\
& \quad=\int_{0}^{T}\left\langle\bar{f}_{1}(t), w(t)\right\rangle d t, \quad \forall w \in L^{2}\left(0, T ; H_{0}^{1}\right), \\
& v(0)=\eta v(T),
\end{aligned}
$$

where $a(\cdot, \cdot)$ is the symmetric bilinear form on $H_{0}^{1} \times H_{0}^{1}$ defined by (21).

Then, we have the following theorem.

Theorem 11. Let $T>0$ and $\left(\bar{H}_{2}\right)-\left(\bar{H}_{6}\right)$ hold. Then, problem (112) has a unique weak solution $v$ such that

$$
\begin{aligned}
& v \in L^{\infty}\left(0, T ; H_{0}^{1}\right), \\
& v_{t} \in L^{2}\left(0, T ; H_{0}^{1}\right) .
\end{aligned}
$$

Proof. The proof consists of several steps.

Step 1 (the Faedo-Galerkin approximation (introduced by Lions [18])). Consider the basis $\left\{w_{j}\right\}$ for $H_{0}^{1}$ as in Lemma 5 . Let $V_{m}$ be the linear space generated by $w_{1}, w_{2}, \ldots, w_{m}$. We consider the following problem. 
Find a function $v_{m}(t)$ in the form (29) satisfying the nonlinear differential equation system $(30)_{1}$ and the $(\eta, T)$ periodic condition:

$$
v_{m}(0)=\eta v_{m}(T) .
$$

We consider an initial value problem given by (30), where $v_{0 m}$ is given in $V_{m}$.

It is clear that, for each $m$, there exists a solution $v_{m}(t)$ in the form (29) which satisfies (30) almost everywhere on $0 \leq t \leq \widetilde{T}_{m}$ for some $\widetilde{T}_{m}, 0<\widetilde{T}_{m} \leq T$. The following a priori estimates allow us to take $\widetilde{T}_{m}=T$ for all $m$.

Step 2 (a priori estimates). Multiplying the $j$ th equation of $(30)_{1}$ by $c_{m j}(t)$ and summing up with respect to $j$, we get

$$
\begin{aligned}
\frac{d}{d t}[ & \left.\left\|v_{m}(t)\right\|_{0}^{2}+\alpha(t)\left\|v_{m x}(t)\right\|_{0}^{2}\right] \\
& +\left(2 \mu(t)-\alpha^{\prime}(t)\right)\left\|v_{m x}(t)\right\|_{0}^{2} \\
& +2\left\langle f\left(v_{m}(t)+\varphi(t)\right), v_{m}(t)\right\rangle \\
= & 2\left\langle\bar{f}_{1}(t), v_{m}(t)\right\rangle .
\end{aligned}
$$

By the same estimates as in Section 3, and with $\varepsilon_{1}>0$, we obtain

$$
\begin{aligned}
& 2\langle f\left.\left(v_{m}(t)+\varphi(t)\right), v_{m}(t)\right\rangle \\
&= 2\left\langle f\left(v_{m}(t)+\varphi(t)\right)-f(\varphi(t)), v_{m}(t)\right\rangle \\
&+2\left\langle f(\varphi(t)), v_{m}(t)\right\rangle \\
& \geq-\left(2 \delta+\varepsilon_{1}\right) \frac{R^{\gamma}}{2}(R-1)^{2}\left\|v_{m x}(t)\right\|_{0}^{2} \\
&-\frac{1}{\varepsilon_{1}}\|f(\varphi(t))\|_{0}^{2} ; \\
& 2\left\langle\bar{f}_{1}(t), v_{m}(t)\right\rangle \leq \frac{1}{\varepsilon_{1}}\left\|\bar{f}_{1}(t)\right\|_{0}^{2}+\varepsilon_{1}\left\|v_{m}(t)\right\|_{0}^{2} \\
& \leq \frac{1}{\varepsilon_{1}}\left\|\bar{f}_{1}(t)\right\|_{0}^{2}+\varepsilon_{1} \frac{R^{\gamma}}{2}(R-1)^{2}\left\|v_{m x}(t)\right\|_{0}^{2} .
\end{aligned}
$$

Hence, it follows from (116) and (117) that

$$
\begin{aligned}
\frac{d}{d t}[ & \left.\left\|v_{m}(t)\right\|_{0}^{2}+\alpha(t)\left\|v_{m x}(t)\right\|_{0}^{2}\right] \\
& +2\left[\bar{\mu}_{*}-\left(\delta+\varepsilon_{1}\right) \frac{R^{\gamma}}{2}(R-1)^{2}\right]\left\|v_{m x}(t)\right\|_{0}^{2} \\
\leq & \frac{1}{\varepsilon_{1}}\left(\left\|\bar{f}_{1}(t)\right\|_{0}^{2}+\|f(\varphi(t))\|_{0}^{2}\right) .
\end{aligned}
$$

By $0<\delta<2 \bar{\mu}_{*} / R^{\gamma}(R-1)^{2}$, choose $\varepsilon_{1}>0$ such that $\tilde{\gamma}=\bar{\mu}_{*}-\left(\delta+\varepsilon_{1}\right)\left(R^{\gamma} / 2\right)(R-1)^{2}>0$.

It is similar to (97); we get

$$
\left\|v_{m x}(t)\right\|_{0}^{2} \geq \beta_{1}\left(\alpha(t)\left\|v_{m x}(t)\right\|_{0}^{2}+\left\|v_{m}(t)\right\|_{0}^{2}\right),
$$

where $\beta_{1}=(1 / 2) \min \left\{1 /\|\alpha\|_{L^{\infty}(0, T)}, 2 / R^{\gamma}(R-1)^{2}\right\}$.
From (118) and (119), it leads to

$$
\begin{aligned}
& \frac{d}{d t}\left[\left\|v_{m}(t)\right\|_{0}^{2}+\alpha(t)\left\|v_{m x}(t)\right\|_{0}^{2}\right] \\
& \quad+2 \beta_{1} \widetilde{\gamma}\left(\left\|v_{m}(t)\right\|_{0}^{2}+\alpha(t)\left\|v_{m x}(t)\right\|_{0}^{2}\right) \leq \bar{f}(t),
\end{aligned}
$$

in which $\bar{f}(t)=\left(1 / \varepsilon_{1}\right)\left[\left\|\bar{f}_{1}(t)\right\|_{0}^{2}+\|f(\varphi(t))\|_{0}^{2}\right]$.

Integrating (120), we have

$$
\begin{aligned}
& \left\|v_{m}(t)\right\|_{0}^{2}+\alpha(t)\left\|v_{m x}(t)\right\|_{0}^{2} \\
& \leq\left(\left\|v_{0 m}\right\|_{0}^{2}+\alpha(0)\left\|v_{0 m x}\right\|_{0}^{2}+\int_{0}^{t} e^{2 \beta_{1} \tilde{\gamma} s} \bar{f}(s) d s\right) \\
& \cdot e^{-2 \beta_{1} \tilde{\gamma} t} \leq \rho^{2}+\left(\left\|v_{0 m}\right\|_{0}^{2}+\alpha(0)\left\|v_{0 m x}\right\|_{0}^{2}-\rho^{2}\right) \\
& \cdot e^{-2 \beta_{1} \tilde{\gamma} t},
\end{aligned}
$$

where $\rho^{2}=\sup _{0 \leq t \leq T} \rho_{1}(t)$, with

$$
\rho_{1}(t)= \begin{cases}\frac{1}{e^{2 \beta_{1} \tilde{\gamma} t}-1} \int_{0}^{t} e^{2 \beta_{1} \tilde{s} s} \bar{f}(s) d s, & 0<t \leq T, \\ \frac{1}{2 \beta_{1} \widetilde{\gamma}} \bar{f}(0), & t=0 .\end{cases}
$$

Therefore, if we choose $v_{0 m}$ such that $\left\|v_{0 m}\right\|_{0}^{2}+\alpha(0)\left\|v_{0 m x}\right\|_{0}^{2}$ $\leq \rho^{2}$, we obtain from (121) that

$$
\begin{array}{r}
\left\|v_{m}(t)\right\|_{0}^{2}+\alpha(t)\left\|v_{m x}(t)\right\|_{0}^{2} \leq \rho^{2}, \\
\text { i.e., } \widetilde{T}_{m}=T
\end{array}
$$

$\forall m$.

Let $\bar{B}_{m}(\rho)$ be a closed ball in the space $V_{m}$ with the norm $v_{0 m} \mapsto\left\|v_{0 m}\right\|_{V_{m}}=\left(\left\|v_{0 m}\right\|_{0}^{2}+\alpha(0)\left\|v_{0 m x}\right\|_{0}^{2}\right)^{1 / 2}$.

By $0<\alpha(0) \leq \alpha(T)$, we obtain

$$
\begin{aligned}
\left\|v_{m}(T)\right\|_{V_{m}}^{2} & =\left\|v_{m}(T)\right\|_{0}^{2}+\alpha(0)\left\|v_{m x}(T)\right\|_{0}^{2} \\
& \leq\left\|v_{m}(T)\right\|_{0}^{2}+\alpha(T)\left\|v_{m x}(T)\right\|_{0}^{2} \leq \rho^{2} .
\end{aligned}
$$

Let us define

$$
\begin{aligned}
\mathscr{F}_{m}: \bar{B}_{m}(\rho) & \longrightarrow \bar{B}_{m}(\rho) \\
v_{0 m} & \longmapsto \mathscr{F}_{m}\left(v_{0 m}\right)=\eta v_{m}(T) .
\end{aligned}
$$

We prove that $\mathscr{F}_{m}$ is a contraction. Let $v_{0 m}, \bar{v}_{0 m} \in$ $\bar{B}_{m}(0, \rho)$ and let $y_{m}(t)=v_{m}(t)-\bar{v}_{m}(t)$, where $v_{m}(t)$ and $\bar{v}_{m}(t)$ are solutions of system $(30)_{1}$ on $[0, T]$ satisfying the initial conditions $v_{m}(0)=v_{0 m}$ and $\bar{v}_{m}(0)=\bar{v}_{0 m}$, respectively. Then, $y_{m}(t)$ satisfies the following differential equation system:

$$
\begin{aligned}
& \left\langle y_{m}^{\prime}(t), w_{j}\right\rangle+\alpha(t) a\left(y_{m}^{\prime}(t), w_{j}\right) \\
& \quad+\mu(t) a\left(y_{m}(t), w_{j}\right) \\
& \quad+\left\langle f\left(v_{m}(t)+\varphi(t)\right)-f\left(\bar{v}_{m}(t)+\varphi(t)\right), w_{j}\right\rangle \\
& =0,
\end{aligned}
$$


where $1 \leq j \leq m$, with initial condition

$$
y_{m}(0)=v_{0 m}-\bar{v}_{0 m} .
$$

By using the same arguments as before, we can show that

$$
\begin{aligned}
& \frac{d}{d t}\left[\left\|y_{m}(t)\right\|_{0}^{2}+\alpha(t)\left\|y_{m x}(t)\right\|_{0}^{2}\right] \\
& \quad+2 \beta_{2}\left[\left\|y_{m}(t)\right\|_{0}^{2}+\alpha(t)\left\|y_{m x}(t)\right\|_{0}^{2}\right] \leq 0,
\end{aligned}
$$

where $\beta_{2}=\beta_{1}\left(\bar{\mu}_{*}-\delta\left(R^{\gamma} / 2\right)(R-1)^{2}\right)>0, \beta_{1}=(1 / 2) \min \{1 /$ $\left.\|\alpha\|_{L^{\infty}(0, T)}, 2 / R^{\gamma}(R-1)^{2}\right\}$.

Integrating inequality (128), we obtain

$$
\begin{aligned}
& \left\|y_{m}(t)\right\|_{0}^{2}+\alpha(t)\left\|y_{m x}(t)\right\|_{0}^{2} \\
& \leq e^{-2 \beta_{2} t}\left(\left\|y_{m}(0)\right\|_{0}^{2}+\alpha(0)\left\|y_{m x}(0)\right\|_{0}^{2}\right) \\
& =e^{-2 \beta_{2} t}\left\|y_{m}(0)\right\|_{V_{m}}^{2}, \quad \forall t \in[0, T] .
\end{aligned}
$$

By $0<\alpha(0) \leq \alpha(T)$, it follows that

$$
\begin{aligned}
\left\|y_{m}(T)\right\|_{V_{m}}^{2} & =\left\|y_{m}(T)\right\|_{0}^{2}+\alpha(0)\left\|y_{m x}(T)\right\|_{0}^{2} \\
& \leq\left\|y_{m}(T)\right\|_{0}^{2}+\alpha(T)\left\|y_{m x}(T)\right\|_{0}^{2} \\
& \leq e^{-2 \beta_{2} T}\left\|y_{m}(0)\right\|_{V_{m}}^{2} \\
& =e^{-2 \beta_{2} T}\left\|v_{0 m}-\bar{v}_{0 m}\right\|_{V_{m}}^{2},
\end{aligned}
$$

or

$$
\begin{aligned}
& \left\|\mathscr{F}_{m}\left(v_{0 m}\right)-\mathscr{F}_{m}\left(\bar{v}_{0 m}\right)\right\|_{V_{m}}=\left\|\eta y_{m}(T)\right\|_{V_{m}} \\
& \quad \leq|\eta| e^{-\beta_{2} T}\left\|v_{0 m}-\bar{v}_{0 m}\right\|_{V_{m}} ;
\end{aligned}
$$

that is, $\mathscr{F}_{m}$ is a contraction.

Therefore, there exists a unique function $v_{0 m} \in \bar{B}_{m}(\rho)$ such that the solution of the initial value problem (30) is a solution of system $(30)_{1}$, (115). This solution satisfies inequality (124) a.e., in $[0, T]$.

On the other hand, multiplying the $j$ th equation of $(30)_{1}$ by $c_{m j}^{\prime}(t)$ and summing up with respect to $j$, afterwards, integrating with respect to the time variable from 0 to $T$, we get after some rearrangements

$$
\begin{aligned}
2 \int_{0}^{T} & \left(\left\|v_{m}^{\prime}(t)\right\|_{0}^{2}+\alpha(t)\left\|v_{m x}^{\prime}(t)\right\|_{0}^{2}\right) d t \\
& +\int_{0}^{T} \frac{d}{d t}\left(\mu(t)\left\|v_{m x}(t)\right\|_{0}^{2}\right) d t \\
& +2 \int_{0}^{T}\left\langle f\left(v_{m}(t)+\varphi(t)\right), v_{m}^{\prime}(t)\right\rangle d t \\
= & \int_{0}^{T} \mu^{\prime}(t)\left\|v_{m x}(t)\right\|_{0}^{2} d t \\
& +2 \int_{0}^{T}\left\langle\bar{f}_{1}(t), v_{m}^{\prime}(t)\right\rangle d t .
\end{aligned}
$$
(32):

We estimate without difficulty the following terms in

$$
\begin{aligned}
& -\int_{0}^{T} \frac{d}{d t}\left(\mu(t)\left\|v_{m x}(t)\right\|_{0}^{2}\right) d t \\
& \quad=-\mu(T)\left\|v_{m x}(T)\right\|_{0}^{2}+\mu(0)\left\|v_{m x}(0)\right\|_{0}^{2} \\
& \quad \leq \mu(0)\left\|v_{m x}(0)\right\|_{0}^{2} \leq \mu(0) \frac{\rho^{2}}{\alpha_{*}} .
\end{aligned}
$$

Note that

$$
\begin{aligned}
& \|\varphi(t)\|_{C^{0}([1, R])} \leq\left|g_{1}(t)\right|+\left|g_{R}(t)\right| \\
& \quad \leq \sup _{0 \leq t \leq T}\left(\left|g_{1}(t)\right|+\left|g_{R}(t)\right|\right), \\
& \left\|v_{m}(t)+\varphi(t)\right\|_{C^{0}([1, R])} \\
& \quad \leq\left\|v_{m}(t)\right\|_{C^{0}([1, R])}+\|\varphi(t)\|_{C^{0}([1, R])} \\
& \quad \leq \sqrt{R-1} \frac{\rho}{\sqrt{\alpha_{*}}}+\sup _{0 \leq t \leq T}\left(\left|g_{1}(t)\right|+\left|g_{R}(t)\right|\right) \\
& \quad=M_{1}(T) .
\end{aligned}
$$

Hence,

$$
\begin{aligned}
2 \int_{0}^{T} & \left\langle f\left(v_{m}(t)+\varphi(t)\right), v_{m}^{\prime}(t)\right\rangle d t \\
\leq & 2 \int_{0}^{T}\left\|f\left(v_{m}(t)+\varphi(t)\right)\right\|_{0}^{2} d t \\
& +\frac{1}{2} \int_{0}^{T}\left\|v_{m}^{\prime}(t)\right\|_{0}^{2} d t \\
\leq & M_{2}(T)+\frac{1}{2} \int_{0}^{T}\left\|v_{m}^{\prime}(t)\right\|_{0}^{2} d t
\end{aligned}
$$

where

$$
M_{2}(T)=2 T\left(\frac{R^{\gamma+1}-1}{\gamma+1}\right) \sup _{|z| \leq M_{1}(T)} f^{2}(z) .
$$

Moreover,

$$
\begin{aligned}
& 2 \int_{0}^{T}\left\langle\bar{f}_{1}(t), v_{m}^{\prime}(t)\right\rangle d t \\
& \quad \leq 2 \int_{0}^{T}\left\|\bar{f}_{1}(t)\right\|_{0}^{2} d t+\frac{1}{2} \int_{0}^{T}\left\|v_{m}^{\prime}(t)\right\|_{0}^{2} d t \\
& \int_{0}^{T} \mu^{\prime}(t)\left\|v_{m x}(t)\right\|_{0}^{2} d t \leq \frac{\rho^{2}}{\alpha_{*}} \int_{0}^{T}\left|\mu^{\prime}(t)\right| d t
\end{aligned}
$$

It follows from (132), (133), (136), and (138) that

$$
\begin{gathered}
\int_{0}^{T}\left\|v_{m}^{\prime}(t)\right\|_{0}^{2} d t+2 \alpha_{*} \int_{0}^{T}\left\|v_{m x}^{\prime}(t)\right\|_{0}^{2} d t \\
\leq \mu(0) \frac{\rho^{2}}{\alpha_{*}}+M_{2}(T)+2 \int_{0}^{T}\left\|\bar{f}_{1}(t)\right\|_{0}^{2} d t \\
+\frac{\rho^{2}}{\alpha_{*}} \int_{0}^{T}\left|\mu^{\prime}(t)\right| d t \leq C_{T},
\end{gathered}
$$


for all $m \in \mathbb{N}$, for all $t \in[0, T]$, where $C_{T}$ always indicates a bound depending on $T$.

Step 3 (the limiting process). By (123) and (139), we deduce that there exists a subsequence of $\left\{v_{m}\right\}$, still denoted by $\left\{v_{m}\right\}$ such that

$$
\begin{aligned}
& v_{m} \longrightarrow v \text { in } L^{\infty}\left(0, T ; H_{0}^{1}\right) \text { weakly*, } \\
& v_{m}^{\prime} \longrightarrow v^{\prime} \text { in } L^{2}\left(0, T ; H_{0}^{1}\right) \text { weakly. }
\end{aligned}
$$

From (115), we obtain

$$
v(0)=\eta v(T) .
$$

Indeed, we prove (141) as follows.

By $\left\|v_{0 m x}\right\| \leq\left(1 / \sqrt{\alpha_{*}}\right)\left\|v_{0 m}\right\|_{V_{m}} \leq \rho / \sqrt{\alpha_{*}}$, and by the imbedding $H_{0}^{1} \hookrightarrow C^{0}(\bar{\Omega})$ being compact, there exists a subsequence of $\left\{v_{0 m}\right\}$, still denoted by $\left\{v_{0 m}\right\}$ such that

$$
\begin{aligned}
& v_{0 m} \longrightarrow \widetilde{v}_{0} \quad \text { in } H_{0}^{1} \text { weakly, } \\
& v_{0 m} \longrightarrow \widetilde{v}_{0} \quad \text { in } C^{0}([1, R]) \text { strongly. }
\end{aligned}
$$

By $v_{m}(t)=v_{m}(0)+\int_{0}^{t} v_{m}^{\prime}(s) d s$, we deduce from (140) and (142) that

$$
v(t)=\widetilde{v}_{0}+\int_{0}^{t} v^{\prime}(s) d s .
$$

This implies

$$
\begin{aligned}
& v(0)=\widetilde{v}_{0}, \\
& v_{0 m} \longrightarrow v(0) \quad \text { in } H_{0}^{1} \text { weakly, } \\
& v_{0 m} \longrightarrow v(0) \quad \text { in } C^{0}([1, R]) \text { strongly. }
\end{aligned}
$$

From (115), we obtain

$$
\begin{aligned}
& \left\langle v_{m}(0), w_{j}\right\rangle=\eta\left\langle v_{m}(T), w_{j}\right\rangle \\
& =\eta\left(\left\langle v_{m}(0), w_{j}\right\rangle+\int_{0}^{T}\left\langle v_{m}^{\prime}(t), w_{j}\right\rangle d t\right),
\end{aligned}
$$

$\forall j \in \mathbb{N}$.

By (140), (145), and (146), it yields

$$
\begin{aligned}
\left\langle v(0), w_{j}\right\rangle & =\eta\left(\left\langle v(0), w_{j}\right\rangle+\int_{0}^{T}\left\langle v^{\prime}(t), w_{j}\right\rangle d t\right) \\
& =\eta\left\langle v(T), w_{j}\right\rangle, \quad \forall j \in \mathbb{N} .
\end{aligned}
$$

Therefore,

$$
v(0)=\eta v(T) .
$$

Therefore, (141) is proved.

Using a compactness lemma ([18], Lions, p. 57), applied to (140), we can extract from the sequence $\left\{v_{m}\right\}$ a subsequence still denoted by $\left\{v_{m}\right\}$, such that

$$
v_{m} \longrightarrow v \quad \text { strongly in } L^{2}\left(Q_{T}\right)
$$

By the Riesz-Fischer theorem, we can extract from $\left\{v_{m}\right\}$ a subsequence still denoted by $\left\{v_{m}\right\}$, such that

$$
v_{m}(x, t) \longrightarrow v(x, t) \quad \text { a.e., }(x, t) \text { in } Q_{T},
$$

Because $f$ is continuous, then

$$
\begin{gathered}
f\left(v_{m}(x, t)+\varphi(x, t)\right) \longrightarrow \\
f(v(x, t)+\varphi(x, t)) \quad \text { a.e., }(x, t) \text { in } Q_{T} .
\end{gathered}
$$

On the other hand,

$$
\begin{array}{r}
\left|f\left(v_{m}(x, t)+\varphi(x, t)\right)\right| \leq \sup _{|z| \leq M_{1}(T)}|f(z)|, \\
\text { a.e., }(x, t) \text { in } Q_{T},
\end{array}
$$

where $M_{1}(T)$ is the constant defined by (135).

Using the dominated convergence theorem, (151) and (152) yield

$$
f\left(v_{m}+\varphi\right) \longrightarrow f(v+\varphi) \quad \text { strongly in } L^{2}\left(Q_{T}\right)
$$

Denote by $\left\{\zeta_{i}, i=1,2, \ldots\right\}$ the orthonormal base in the real Hilbert space $L^{2}(0, T)$. The set $\left\{\zeta_{i} w_{j}, i, j=1,2, \ldots\right\}$ forms an orthonormal base in $L^{2}\left(0, T ; H_{0}^{1}\right)$. From $(30)_{1}$ we have

$$
\begin{aligned}
& \int_{0}^{T}\left\langle v_{m}^{\prime}(t), w_{j} \zeta_{i}(t)\right\rangle d t \\
& \quad+\int_{0}^{T} \alpha(t) a\left(v_{m}^{\prime}(t), w_{j} \zeta_{i}(t)\right) d t \\
& \quad+\int_{0}^{T} \mu(t) a\left(v_{m}(t), w_{j} \zeta_{i}(t)\right) d t \\
& \quad+\int_{0}^{T}\left\langle f\left(v_{m}(t)+\varphi(t)\right), w_{j} \zeta_{i}(t)\right\rangle d t \\
& =\int_{0}^{T}\left\langle\bar{f}_{1}(t), w_{j} \zeta_{i}(t)\right\rangle d t,
\end{aligned}
$$

for all $i, j, 1 \leq j \leq m, i \in \mathbb{N}$.

For $i, j$ fixed, passing to the limit in (154) by (140) and (153), we get

$$
\begin{aligned}
& \int_{0}^{T}\left\langle v^{\prime}(t), w_{j} \zeta_{i}(t)\right\rangle d t \\
& \quad+\int_{0}^{T} \alpha(t) a\left(v^{\prime}(t), w_{j} \zeta_{i}(t)\right) d t \\
& \quad+\int_{0}^{T} \mu(t) a\left(v(t), w_{j} \zeta_{i}(t)\right) d t \\
& \quad+\int_{0}^{T}\left\langle f(v(t)+\varphi(t)), w_{j} \zeta_{i}(t)\right\rangle d t \\
&=\int_{0}^{T}\left\langle\bar{f}_{1}(t), w_{j} \zeta_{i}(t)\right\rangle d t .
\end{aligned}
$$


Note that (155) holds for every $i, j \in \mathbb{N}$; that is, the equality

$$
\begin{aligned}
\int_{0}^{T}\left\langle v^{\prime}(t), w(t)\right\rangle d t+\int_{0}^{T} \alpha(t) a\left(v^{\prime}(t), w(t)\right) d t \\
\quad+\int_{0}^{T} \mu(t) a(v(t), w(t)) d t \\
\quad+\int_{0}^{T}\langle f(v(t)+\varphi(t)), w(t)\rangle d t \\
=\int_{0}^{T}\left\langle\bar{f}_{1}(t), w(t)\right\rangle d t, \quad \forall w \in L^{2}\left(0, T ; H_{0}^{1}\right),
\end{aligned}
$$

is fulfilled.

Step 4 (uniqueness of the solutions). Let $v_{1}$ and $v_{2}$ be two solutions of (113). Then $v=v_{1}-v_{2}$ satisfies the following problem:

$$
\begin{aligned}
& \int_{0}^{T}\left\langle v^{\prime}(t), w(t)\right\rangle d t+\int_{0}^{T} \alpha(t) a\left(v^{\prime}(t), w(t)\right) d t \\
& +\int_{0}^{T} \mu(t) a(v(t), w(t)) d t \\
& +\int_{0}^{T}\left\langle f\left(v_{1}(t)+\varphi(t)\right)\right. \\
& \left.-f\left(v_{2}(t)+\varphi(t)\right), w(t)\right\rangle d t=0, \\
& v(0)=\eta v(T), \quad \forall w \in L^{2}\left(0, T ; H_{0}^{1}\right), \\
& v \in L^{\infty}\left(0, T ; H_{0}^{1}\right), \quad v_{t} \in L^{2}\left(0, T ; H_{0}^{1}\right) .
\end{aligned}
$$

Taking $w=2 v$ in $(157)_{1}$ and using $(157)_{2}$, we get

$$
\begin{aligned}
& \int_{0}^{T}\left(2 \mu(t)-\alpha^{\prime}(t)\right)\left\|v_{x}(t)\right\|_{0}^{2} d t+\int_{0}^{T} \frac{d}{d t}\|v(t)\|_{0}^{2} d t \\
& \quad+\int_{0}^{T} \frac{d}{d t}\left(\alpha(t)\left\|v_{x}(t)\right\|_{0}^{2}\right) d t \\
& \quad+2 \int_{0}^{T}\left\langle f\left(v_{1}(t)+\varphi(t)\right)\right. \\
& \left.\quad-f\left(v_{2}(t)+\varphi(t)\right), v(t)\right\rangle d t=0 .
\end{aligned}
$$

By $2 \mu(t)-\alpha^{\prime}(t) \geq \bar{\mu}_{*}>0$, we have

$$
\begin{gathered}
\int_{0}^{T}\left(2 \mu(t)-\alpha^{\prime}(t)\right)\left\|v_{x}(t)\right\|_{0}^{2} d t \\
\geq 2 \bar{\mu}_{*} \int_{0}^{T}\left\|v_{x}(t)\right\|_{0}^{2} d t .
\end{gathered}
$$

On the other hand,

$$
\begin{aligned}
& \int_{0}^{T} \frac{d}{d t}\|v(t)\|_{0}^{2} d t=\|v(T)\|_{0}^{2}-\|v(0)\|_{0}^{2} \\
& \quad=\left(1-\eta^{2}\right)\|v(T)\|_{0}^{2} \geq 0 \\
& \int_{0}^{T} \frac{d}{d t}\left(\alpha(t)\left\|v_{x}(t)\right\|_{0}^{2}\right) d t \\
& \quad=\alpha(t)\left\|v_{x}(T)\right\|_{0}^{2}-\alpha(0)\left\|v_{x}(0)\right\|_{0}^{2} \\
& \quad \geq \alpha(0)\left(1-\eta^{2}\right)\left\|v_{x}(T)\right\|_{0}^{2} \geq 0
\end{aligned}
$$

Hence,

$$
\begin{aligned}
2 \bar{\mu}_{*} & \int_{0}^{T}\left\|v_{x}(t)\right\|_{0}^{2} d t \leq-2 \int_{0}^{T}\left\langle f\left(v_{1}(t)+\varphi(t)\right)\right. \\
- & \left.f\left(v_{2}(t)+\varphi(t)\right), v(t)\right\rangle d t \\
& \leq 2 \delta \int_{0}^{T}\|v(t)\|_{0}^{2} d t \leq 2 \delta \frac{R^{\gamma}}{2}(R-1)^{2} \\
& \cdot \int_{0}^{T}\left\|v_{x}(t)\right\|_{0}^{2} d t .
\end{aligned}
$$

By $0<\delta<2 \bar{\mu}_{*} / R^{\gamma}(R-1)^{2}$, implying $\delta\left(R^{\gamma} / 2\right)(R-1)^{2}<$ $\bar{\mu}_{*}$, we deduce from (161) that $\int_{0}^{T}\left\|v_{x}(t)\right\|_{0}^{2} d t=0$; that is, $v=$ $v_{1}-v_{2}=0$.

This completes the proof of Theorem 11 .

\section{Competing Interests}

The authors declare that they have no competing interests.

\section{Authors' Contributions}

All authors contributed equally to this article. They read and approved the final manuscript.

\section{References}

[1] A. B. Al'shin, M. O. Korpusov, and A. G. Sveshnikov, Blow-Up in Nonlinear Sobolev Type Equations, vol. 15 of De Gruyter Series in Nonlinear Analysis and Applications, Walter de Gruyter \& Co, Berlin, Germany, 2011.

[2] R. W. Carroll and R. E. Showalter, Singular and Degenerate Cauchy Problems, vol. 127 of Mathematics in Science and Engineering, Academic Press, Harcourt Brace Jovanovich, New York, NY, USA, 1976.

[3] R. E. Showalter and T. W. Ting, "Asymptotic behavior of solutions of pseudo-parabolic partial differential equations," Annali di Matematica Pura ed Applicata. Serie Quarta, vol. 90, pp. 241258, 1971.

[4] R. E. Showalter, "Existence and representation theorems for a semilinear Sobolev equation in Banach space," SIAM Journal on Mathematical Analysis, vol. 3, pp. 527-543, 1972.

[5] A. Mahmood, N. A. Khan, C. Fetecau, M. Jamil, and Q. Rubbab, "Exact analytic solutions for the flow of second grade fluid between two longitudinally oscillating cylinders," Journal of Prime Research in Mathematics, vol. 5, pp. 192-204, 2009. 
[6] S. Asghar, T. Hayat, and P. D. Ariel, "Unsteady Couette flows in a second grade fluid with variable material properties," Communications in Nonlinear Science and Numerical Simulation, vol. 14, no. 1, pp. 154-159, 2009.

[7] T. Hayat, M. Khan, A. M. Siddiqui, and S. Asghar, "Transient flows of a second grade fluid," International Journal of NonLinear Mechanics, vol. 39, no. 10, pp. 1621-1633, 2004.

[8] T. Hayat, M. Khan, M. Ayub, and A. M. Siddiqui, "The unsteady Couette flow of a second grade fluid in a layer of porous medium," Archives of Mechanics, vol. 57, no. 5, pp. 405-416, 2005.

[9] T. Hayat, M. Khan, and M. Ayub, "Some analytical solutions for second grade fluid flows for cylindrical geometries," Mathematical and Computer Modelling, vol. 43, no. 1-2, pp. 16-29, 2006.

[10] T. Hayat, M. Hussain, and M. Khan, "Hall effect on flows of an Oldroyd-B fluid through porous medium for cylindrical geometries," Computers and Mathematics with Applications, vol. 52, no. 3-4, pp. 269-282, 2006.

[11] T. Hayat, M. Sajid, and M. Ayub, "On explicit analytic solution for MHD pipe flow of a fourth grade fluid," Communications in Nonlinear Science and Numerical Simulation, vol. 13, no. 4, pp. 745-751, 2008.

[12] N. T. Long and A. P. N. Dinh, "On a nonlinear parabolic equation involving Bessel's operator associated with a mixed inhomogeneous condition," Journal of Computational and Applied Mathematics, vol. 196, no. 1, pp. 267-284, 2006.

[13] L. T. P. Ngoc, N. Van Y, A. P. N. Dinh, and N. T. Long, "On a nonlinear heat equation associated with Dirichlet-Robin conditions," Numerical Functional Analysis and Optimization, vol. 33, no. 2, pp. 166-189, 2012.

[14] S. Cordier, L. X. Truong, N. T. Long, and A. P. N. Dinh, "Large time behavior of differential equations with drifted periodic coefficients modeling carbon storage in soil," Applied Mathematics and Computation, vol. 218, no. 9, pp. 5641-5654, 2012.

[15] L. T. P. Ngoc, T. T. Nhan, T. M. Thuyet, and N. T. Long, "On the nonlinear pseudoparabolic equation with the mixed inhomogeneous condition," Boundary Value Problems, vol. 2016, article 137, 2016.

[16] L. X. Truong, L. T. Ngoc, C. H. Hoa, and N. T. Long, “On a system of nonlinear wave equations associated with the helical flows of Maxwell fluid," Nonlinear Analysis: Real World Applications, vol. 12, no. 6, pp. 3356-3372, 2011.

[17] R. E. Showater, "Hilbert space methods for partial differential equations," Electronic Journal of Differential Equations, Monograph 01, 1994.

[18] J. L. Lions, Quelques Méthodes de Résolution des Problèmes Aux Limites Nonlinéaires, Dunod; Gauthier-Villars, Paris, France, 1969. 


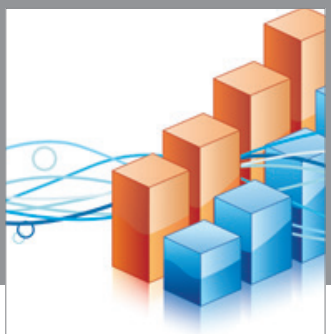

Advances in

Operations Research

vatem alat4

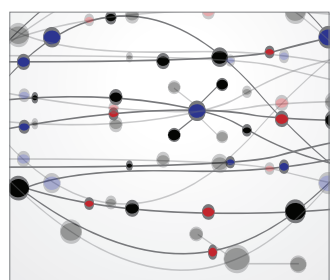

\section{The Scientific} World Journal
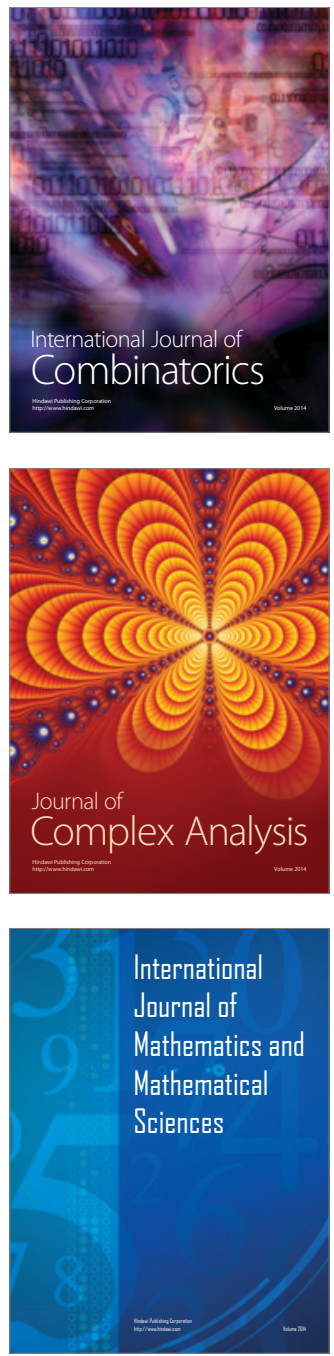
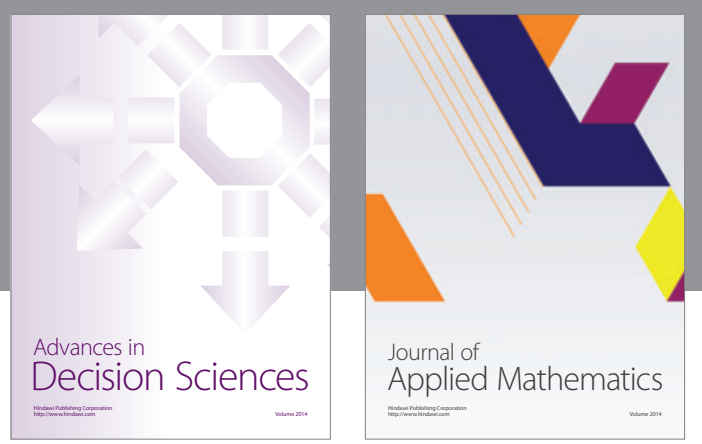

Algebra

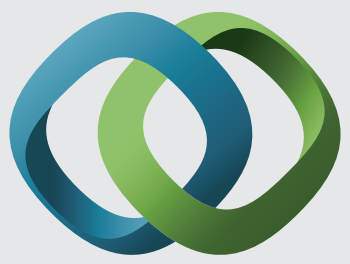

\section{Hindawi}

Submit your manuscripts at

http://www.hindawi.com
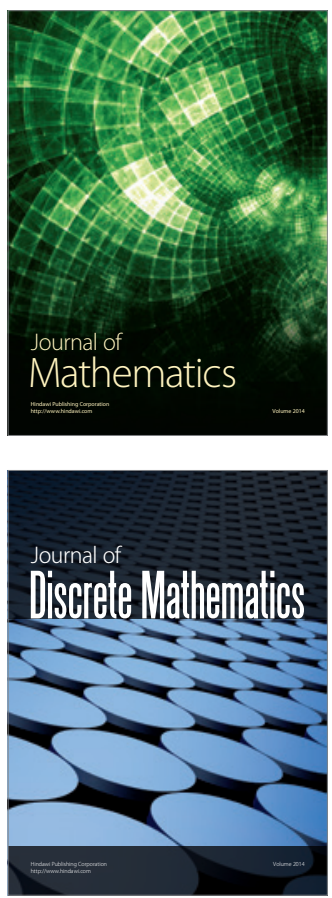

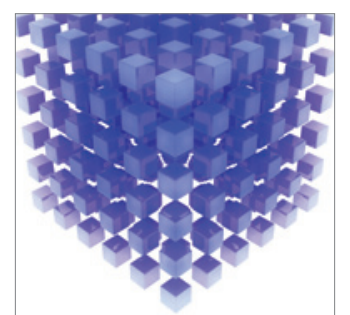

Mathematical Problems in Engineering
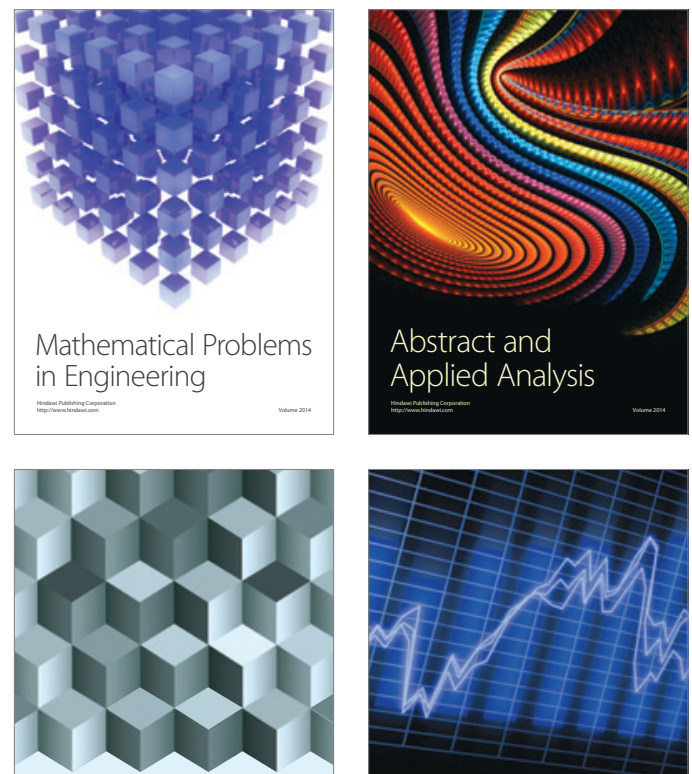

Journal of

Function Spaces

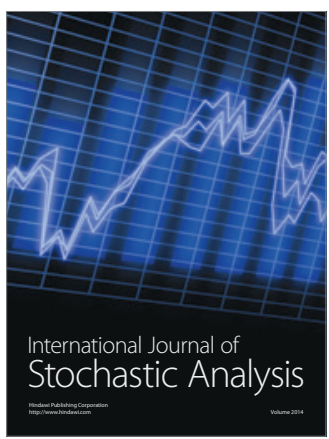

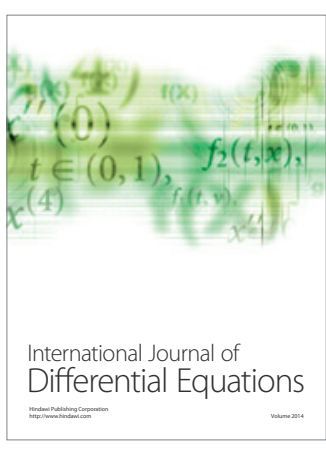
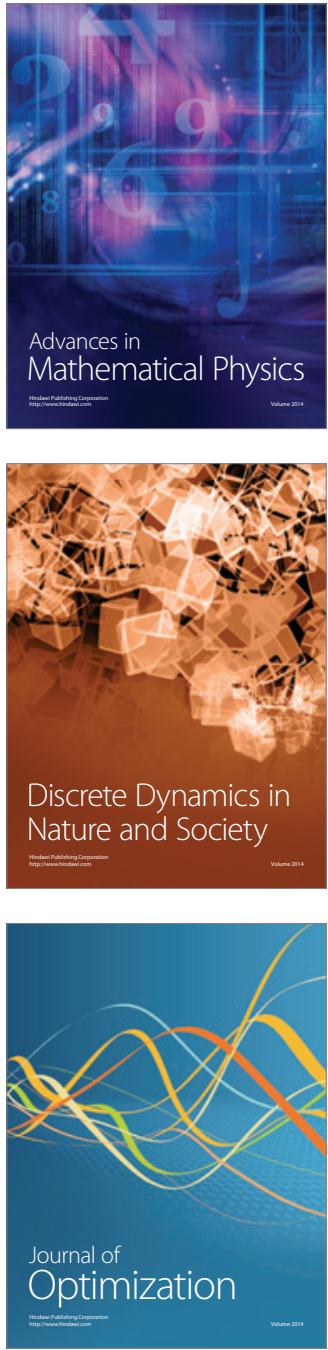